\title{
Breather solutions of a fourth-order nonlinear Schrödinger equation in the degenerate, soliton, and rogue wave limits
}

\author{
Amdad Chowdury and Wieslaw Krolikowski \\ Science Program, Texas A\&M University at Qatar, Doha, Qatar \\ N. Akhmediev \\ Optical Sciences Group, Research School of Physics and Engineering, Australian National University, Canberra, ACT 2600, Australia
}

(Received 25 July 2017; published 18 October 2017)

\begin{abstract}
We present one- and two-breather solutions of the fourth-order nonlinear Schrödinger equation. With several parameters to play with, the solution may take a variety of forms. We consider most of these cases including the general form and limiting cases when the modulation frequencies are 0 or coincide. The zero-frequency limit produces a combination of breather-soliton structures on a constant background. The case of equal modulation frequencies produces a degenerate solution that requires a special technique for deriving. A zero-frequency limit of this degenerate solution produces a rational second-order rogue wave solution with a stretching factor involved. Taking, in addition, the zero limit of the stretching factor transforms the second-order rogue waves into a soliton. Adding a differential shift in the degenerate solution results in structural changes in the wave profile. Moreover, the zero-frequency limit of the degenerate solution with differential shift results in a rogue wave triplet. The zero limit of the stretching factor in this solution, in turn, transforms the triplet into a singlet plus a low-amplitude soliton on the background. A large value of the differential shift parameter converts the triplet into a pure singlet.
\end{abstract}

DOI: 10.1103/PhysRevE.96.042209

\section{INTRODUCTION}

Modulation instability (MI) is the initial process of growth of a small periodic perturbation on a carrier wave or a continuous wave background leading to the formation of patterns and structures in nature. This fundamental mechanism of pattern formation has been observed in nonlinear optics [1], hydrodynamics [2], plasmas [3], and biology [4]. Early work on MI that can be traced back to the 50s [4] 60s [1], and 70s [3] focused primarily on the initial, linear stage of instability. However, considering only the initial stage cannot answer the fundamental questions: What happens afterwards? and What is the final stage of the complex process that starts with MI? Answers to these questions can be found using numerical simulations $[5,6]$. However, exact solutions remain the main tool when we are dealing with integrable equations. In this regard, the discovery of Akhmediev breathers $[7,8]$ was a significant step in the present state of the matter. The latter is a periodic solution of the nonlinear Schrödinger equation (NLSE) that is a natural continuation of MI.

The generation of trains of picosecond pulses in an optical fiber and their evolution are one of the practical aspects of the periodic solution of the NLSE [8-10]. The universal nature of this periodic solution is presently well established and the number of theoretical and experimental studies in connection with various nonlinear media is growing $[9,10]$. The significance of this solution lies also in its intimate connection with the phenomenon of Fermi-Pasta-Ulam recurrence [11]. Most recently, this breather solution appeared to be useful in connection with the formation of rogue waves $[12,13]$.

These ideas can be expanded to a wider range of integrable equations. Indeed, the basic form of the NLSE only includes the lowest-order dispersion and the lowest-order nonlinear term. Higher-order effects are essential when describing ultrashort pulses generated as a result of the MI [14-17]. Each of the higher-order terms makes a highly specific contribution to pulse propagation [18]. However, only special combinations of them in the fiber lead to integrable equations. Despite being very specific, these cases deserve attention because of the exact solutions that can be derived for them.

In this work, we present breather solutions for the fourthorder nonlinear Schrödinger equation [19,20]. Namely, we are dealing with

$$
\begin{aligned}
i \psi_{x} & +\alpha_{2}\left(\psi_{t t}+2 \psi|\psi|^{2}\right)+\alpha_{4}\left(\psi_{t t t t}+8|\psi|^{2} \psi_{t t}+4 \psi\left|\psi_{t}\right|^{2}\right. \\
& \left.+6 \psi_{t}^{2} \psi^{*}+2 \psi^{2} \psi_{t t}^{*}+6 \psi|\psi|^{4}\right)=0
\end{aligned}
$$

where $\psi(x, t)$ may represent, e.g., in the context of optics, the amplitude of the electric field of the electromagnetic wave. The coefficient $\alpha_{2}$ in Eq. (1) scales the terms of the standard NLSE, while the coefficient $\alpha_{4}$ scales the fourth-order terms. Porsezian at el. derived this equation in connection with integrability aspects of the one-dimensional Heisenberg spin chain problem [21]. Recently, the NLSE has been extended to include an infinite number of terms [22-27]. Coefficients $\alpha_{i}$ representing the higher-order terms in this hierarchy are arbitrary, in contrast to earlier works [28], where they are small parameters. Equation (1) is a member of this hierarchy that includes the second- and fourth-order operators.

Breathers and rogue wave solutions of Eq. (1) have been found in Refs. [20,22,23,29]. Here, we concentrate on special cases, transformations, and degenerate solutions which follow from the general solution. These special cases are far from trivial and deserve separate study. For example, degenerate solutions of the NLSE have been discussed in detail in Ref. [30]. In contrast to the NLSE, Eq. (1) has free parameters, which make such a study much more involved. Higher-order terms add more features to solutions. One aspect of the degenerate solutions presented here is that breathers and rogue waves can be transformed into solitons [31-33]. We reveal a variety of these breather-soliton interactions that do not exist in the case of the standard NLSE. 


\section{FIRST-ORDER BREATHER SOLUTION}

We start by analyzing the fundamental breather solution and some particular cases. The first-order breather solution to Eq. (1) is given by

$$
\psi_{1}=\left(\frac{\kappa\left\{\kappa^{2} \cosh \left[V_{H} x_{s 1}\right]+2 i \delta \sinh \left[V_{H} x_{s 1}\right]\right\}}{2\left\{\kappa \cosh \left[V_{H} x_{s 1}\right]-\delta \cos \left[\kappa t_{s 1}\right]\right\}}-1\right) e^{i \omega x},
$$

where $V_{H}=2 \delta\left[\alpha_{2}-\alpha_{4}\left(\kappa^{2}-6\right)\right], x_{s j}=\left(x-x_{j}\right)$, and $t_{s j}=$ $\left(t-t_{j}\right)$. Parameters $x_{j}$ and $t_{j}$ are translations along the $x$ and $t$ axes. This solution is a direct continuation of the Akhmediev breather $(\mathrm{AB})$ solution for the NLSE $[7,8]$. The indices $j$ in (2) are introduced for convenience of further analysis.

Roughly speaking, the first-order breather solution is analogous to a one-soliton solution of the fundamental NLSE, while the second-order solution presented below is analogous to a two-soliton solution. More specifically, the second-order solution can be considered the nonlinear superposition of two first-order ones.

When dealing with two-breather solutions, we can take $j=1,2$ for each of them. For the first-order breather solution with $j=1$, we use $x_{s 1}=\left(x-x_{1}\right)$ and $t_{s 1}=\left(t-t_{1}\right)$. In the exponent, $\omega=2\left(\alpha_{2}+3 \alpha_{4}\right)$. The parameter $\kappa$ is the modulation frequency of the breather, and $\delta=\frac{1}{2} \kappa \sqrt{4-\kappa^{2}}$ is the growth rate of the modulation leading to Eq. (2). With $\alpha_{4}=0$, Eq. (1) reduces to the NLSE and the breather solution, Eq. (2), reduces to the NLSE AB solution [8].

There are particular cases of this solution that distinguish it from the NLSE AB. This happens because Eq. (1) has free parameters that the NLSE is missing. The coefficient $V_{H}$ in the hyperbolic functions in Eq. (2) plays an important role in reshaping the structure of the breather solution. For instance, if we take $V_{H}=0$, the $\mathrm{AB}$ turns into a $t$ periodic solution. Namely, when $\alpha_{2}=\alpha_{4}\left(\kappa^{2}-6\right)$, the argument of the hyperbolic function $V_{H}$ becomes 0 and Eq. (2) turns into

$$
\psi_{1}=\left(\frac{\kappa^{2}}{2-\sqrt{4-\kappa^{2}} \cos \left[\kappa t_{s 1}\right]}-1\right) e^{i \omega^{\prime} x},
$$

where $\omega^{\prime}=2 \alpha_{4}\left(\kappa^{2}-3\right)$. This is a zero-velocity periodic solution with frequency $\kappa$ within the interval $0<\kappa<2$. Now, taking the limit $\kappa \rightarrow 0$, Eq. (3) reduces to a rational solution:

$$
\psi_{1}=\left(\frac{4}{1+4 t_{s 1}^{2}}-1\right) e^{-6 i \alpha_{4} x}
$$

This can be considered a one-soliton solution on a background propagating along the $x$ direction.

Another form of the solution, (2), appears when $\kappa$ is imaginary. If we assume $\kappa=i p$ in Eq. (2), then the growth rate $\delta=i g$, where $g=p \sqrt{4+p^{2}} / 2$. Using the conversion formulas of hyperbolic to trigonometric functions, $\sinh (z) \rightarrow$ $-i \sin (i z)$ and $\cosh [z] \rightarrow \cos (i z)$, we convert $V_{H} \rightarrow V_{T}$. The latter coefficient enters the argument of trigonometric functions. Now taking the value of $V_{T}=2 g\left[\alpha_{2}+\alpha_{4}\left(6+p^{2}\right)\right]$, the new solution becomes

$$
\psi_{1}=\left(\frac{-p\left\{p^{2} \cos \left[V_{T} x_{s 1}\right]+2 i g \sin \left[V_{T} x_{s 1}\right]\right\}}{2\left\{p \cos \left[V_{T} x_{s 1}\right]-2 g \cosh \left[p t_{s 1}\right]\right\}}-1\right) e^{i \omega x} .
$$

This is the so-called 'Kuznetsov' [34] or 'Ma' [35] (KM) soliton solution on a background that is periodic in time and localized in space. In a particular case, when the argument of the trigonometric function $V_{T}=0$, with the condition $\alpha_{2}=$ $-\alpha_{4}\left(6+p^{2}\right)$, we have, from Eq. (5),

$$
\psi_{1}=\left(\frac{p^{2}}{\sqrt{4+p^{2}} \cosh \left[p t_{s 1}\right]-2}-1\right) e^{-2 i \alpha_{4}\left(p^{2}+3\right) x} .
$$

However, in contrast to Eq. (3), which is periodic in $t$, Eq. (6) is clearly a one-soliton solution on a background. In the limit $p \rightarrow 0$, Eq. (6) reduces to the soliton solution, (4). However, a direct limit of Eq. (2) with $\kappa \rightarrow 0$ or Eq. (5) with $p \rightarrow 0$ will give us the rational Peregrine soliton, which is localized in both $x$ and $t$.

The general expression for the solution of any order has the form [20]

$$
\psi_{n}(x, t)=\left((-1)^{n}+\frac{G_{n}+i H_{n}}{D_{n}}\right) e^{i \omega x},
$$

where $n$ indicates the order of the solution. The functions $G_{n}(x, t), H_{n}(x, t)$, and $D_{n}(x, t)$ are real. For each particular case, these expressions are provided in the next sections. For example, for a first-order rational solution with $n=1$, the polynomials $G_{1}, H_{1}$, and $D_{1}$ are given by

$$
\begin{aligned}
& G_{1}=4, \\
& H_{1}=16 B x_{s 1}, \\
& D_{1}=1+4 t_{s 1}^{2}+16 B^{2} x_{s 1}^{2},
\end{aligned}
$$

while $\omega=2\left(\alpha_{2}+3 \alpha_{4}\right)$. Here the stretching factor $B=\left(\alpha_{2}+\right.$ $6 \alpha_{4}$ ). If $B \rightarrow 0$ when $\alpha_{2}=-6 \alpha_{4}$, clearly the first-order rogue wave solution reduces to a rational soliton, (4). On the other hand, if $\alpha_{4}=0$, the first-order solution of Eq. (1) reduces to a standard rogue wave solution of the NLSE [13].

\section{SECOND-ORDER BREATHER SOLUTION}

Two-breather solutions of Eq. (1) in various forms have been studied in Refs. [29,36]. However, the results were incomplete and require more detailed analysis. Several important special cases were missing, which we augment here. The technique that we employ to derive the solution is described in Ref. [10]. It is based on two eigenvalues $\lambda_{j}$ expressed in terms of the modulation frequencies of the two breathers $\kappa_{j}=2 \sqrt{1+\lambda_{j}^{2}}$, where $j=1,2$ is the number of breathers. With $n=2$ in the general expression, (7), the two-breather solution of Eq. (1) is given by

$$
\begin{aligned}
G_{2}= & \frac{\kappa_{1}^{2}-\kappa_{2}^{2}}{\kappa_{1} \kappa_{2}}\left\{-\delta_{2} \kappa_{1}^{3} \cos \left(t_{\mathrm{s} 2} \kappa_{2}\right) \cosh \left(V_{H 1} x_{\mathrm{s} 1}\right)\right. \\
& +\kappa_{2} \cosh \left(V_{H 2} x_{\mathrm{s} 2}\right)\left[\delta_{1} \kappa_{2}^{2} \cos \left(t_{\mathrm{s} 1} \kappa_{1}\right)\right. \\
& \left.\left.+\kappa_{1}\left(\kappa_{1}^{2}-\kappa_{2}^{2}\right) \cosh \left(V_{H 1} x_{\mathrm{s} 1}\right)\right]\right\}, \\
H_{2}= & \frac{2\left(\kappa_{1}^{2}-\kappa_{2}^{2}\right)}{\kappa_{1} \kappa_{2}}\left\{\delta _ { 2 } \kappa _ { 2 } \operatorname { s i n h } ( V _ { H 2 } x _ { \mathrm { s } 2 } ) \left[\delta_{1} \cos \left(t_{\mathrm{s} 1} \kappa_{1}\right)\right.\right. \\
& \left.-\kappa_{1} \cosh \left(V_{H 1} x_{\mathrm{s} 1}\right)\right]+\delta_{1} \kappa_{1} \sinh \left(V_{H 1} x_{\mathrm{s} 1}\right) \\
& \left.\times\left[\kappa_{2} \cosh \left(V_{H 2} x_{\mathrm{s} 2}\right)-\delta_{2} \cos \left(t_{\mathrm{s} 2} \kappa_{2}\right)\right]\right\},
\end{aligned}
$$




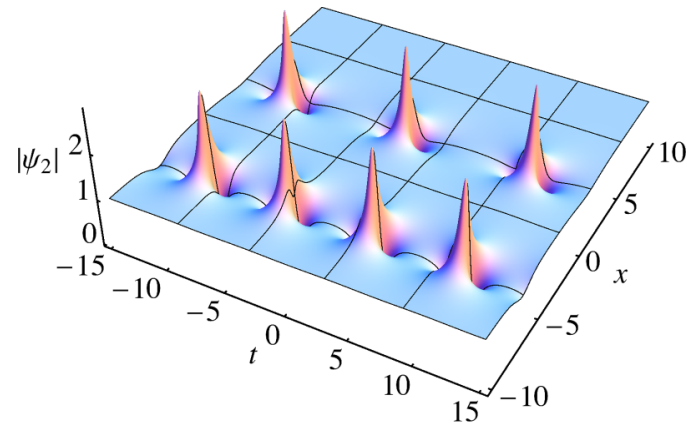

(a)

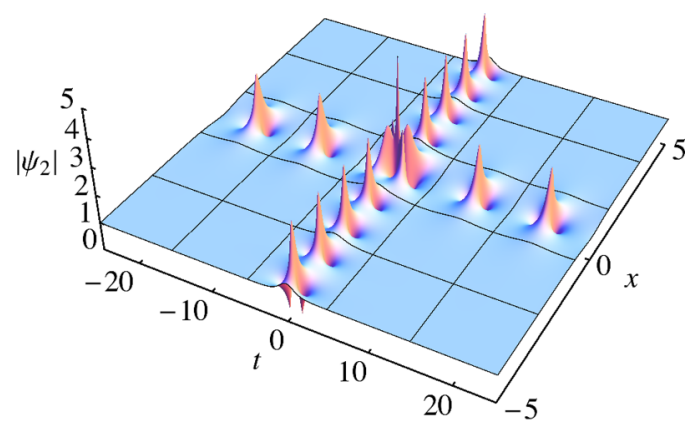

(b)

FIG. 1. Two-breather solution, (9), for two cases: (a) $\kappa_{1}=0.6$, $\kappa_{2}=0.9, x_{1}=5$, and $x_{2}=-5$; (b) $\kappa_{1}=0.7$ and $\kappa_{2}=1.1 i$. In each case, $\alpha_{2}=1 / 2$ and $\alpha_{4}=1 / 16$.

$$
\begin{aligned}
D_{1}= & \frac{1}{\kappa_{1} \kappa_{2}}\left\{\kappa _ { 1 } \operatorname { c o s h } ( V _ { H 1 } x _ { \mathrm { s } 1 } ) \left\{2 \delta_{2}\left(\kappa_{1}^{2}-\kappa_{2}^{2}\right) \cos \left(t_{\mathrm{s} 2} \kappa_{2}\right)\right.\right. \\
& \left.+\kappa_{2} \cosh \left(V_{H 2} x_{\mathrm{s} 2}\right)\left[\kappa_{1}^{2}\left(\kappa_{2}^{2}-2\right)-2 \kappa_{2}^{2}\right]\right\} \\
& +2 \delta_{1}\left[\delta_{2}\left(\kappa_{1}^{2}+\kappa_{2}^{2}\right) \cos \left(t_{\mathrm{s} 1} \kappa_{1}\right) \cos \left(t_{\mathrm{s} 2} \kappa_{2}\right)\right. \\
& +\kappa_{2}\left(2 \delta _ { 2 } \kappa _ { 1 } \left[\sin \left(t_{\mathrm{s} 1} \kappa_{1}\right) \sin \left(t_{\mathrm{s} 2} \kappa_{2}\right)\right.\right. \\
& \left.+\sinh \left(V_{H 1} x_{\mathrm{s} 1}\right) \sinh \left(V_{H 2} x_{\mathrm{s} 2}\right)\right] \\
& \left.\left.\left.+\left(\kappa_{2}^{2}-\kappa_{1}^{2}\right) \cos \left(t_{\mathrm{s} 1} \kappa_{1}\right) \cosh \left(V_{H 2} x_{\mathrm{s} 2}\right)\right)\right]\right\},
\end{aligned}
$$

where $\quad \delta_{j}=\kappa_{j} \sqrt{4-\kappa_{j}^{2}} / 2, \quad V_{H j}=2 \delta_{j}\left[\alpha_{2}-\alpha_{4}\left(\kappa_{j}^{2}-6\right)\right]$, $x_{s j}=\left(x-x_{j}\right)$, and $t_{s j}=\left(t-t_{j}\right)$, with $x_{j}$ and $t_{j}$ being translations along the $x$ and $t$ axes. Modulation frequencies $\kappa_{1}$ and $\kappa_{2}$ are two independent free parameters that play the key role in mutual interaction between the breathers.

One example of the two-breather solution is shown in Fig. 1(a). Here, the first breather, with modulation frequency $\kappa_{1}=0.6$, is located on the $x=5$ line, while the second one, with frequency $\kappa_{2}=0.9$, arrives with a time delay of $x_{2}=-5$.

Depending on the parameters $\kappa$, Eq. (9) may represent both $\mathrm{AB}$ and KM solitons. For example, in Fig. 1(b), the first breather, with $\kappa_{1}=0.7$, is an AB located on the $x=0$ line, while the second one is actually a $\mathrm{KM}$ soliton with $\kappa_{2}=1.1 i$. It is orthogonal to the $\mathrm{AB}$. When $\alpha_{4}=0$, the solution reduces to the standard NLSE solution presented in Refs. [8,30]. The two-breather solution for a higher-order NLSE with quintic terms has been given in Ref. [37].

How the two breathers interact depends on the ratio of their modulation frequencies $\kappa_{1}$ and $\kappa_{2}$. Breather interaction

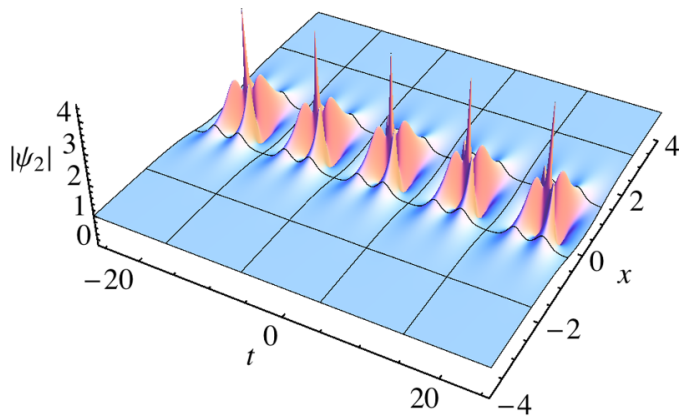

(a)

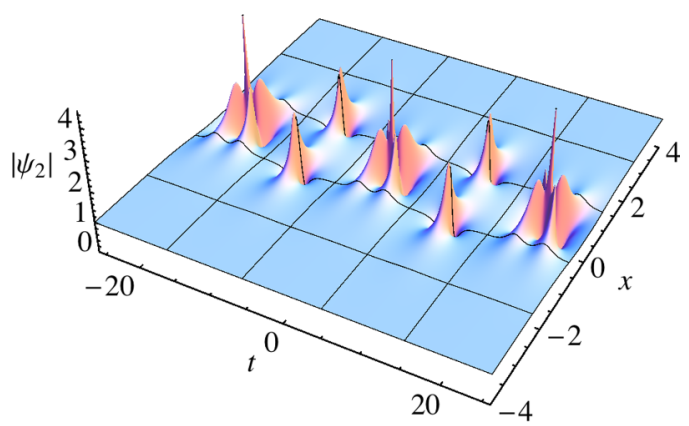

(b)

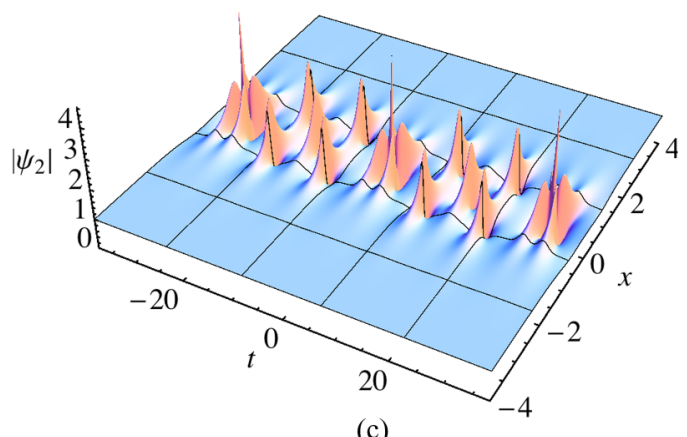

(c)

FIG. 2. Two-breather solution, (9), with integer ratios of modulation frequencies: (a) $\kappa_{1}: \kappa_{2}=2: 1$, (b) $\kappa_{1}: \kappa_{2}=3: 2$, and (c) $\kappa_{1}: \kappa_{2}=5: 3$. Parameters used for plotting are $\alpha_{2}=\frac{1}{2}, \alpha_{4}=\frac{1}{4}, \kappa_{2}=0.6$.

descriptions have been given in Refs. [8,30,37]. Here we give an alternative explanation of similar scenarios. To set the rule precisely, let us define the relation between them as

$$
\kappa_{1}: \kappa_{2}=a: b
$$

where $a$ and $b$ are two integers defining the ratio between $\kappa_{1}$ and $\kappa_{2}$. When $a=2$ and $b=1$, the two-breather solution creates the periodic sequence of second-order rogue wave profiles shown in Fig. 2(a). In turn, each second-order rogue wave profile is made up of three individual first-order rogue waves [38].

Within each periodic sequence, the first-order components are organized following specific rules. First, the total number of first-order rogue waves in each period is the sum of the integers defining the ratios of the modulation frequencies $\kappa_{1}$ and $\kappa_{2}$, that is, $a+b$. Second, of the total number, three of them always merge to form a second-order profile as shown 
in Fig. 2(a). The rest are located symmetrically around the central line $x=0$. Note also that the ratio of $a$ and $b$ should be chosen such that the modulation frequency remains within the instability band $0<\kappa_{j}<2$.

For example, in Fig. 2(a), the modulation frequencies $\kappa_{1}$ and $\kappa_{2}$ are related as $a: b=2: 1$. Therefore, when $\kappa_{2}=$ $0.6, \kappa_{1}=(a / b) \kappa_{2}=1.2$. Thus, the total number of firstorder rogue wave components in each period in this case is $a+b=3$. All three are merged, comprising a periodic sequence of second-order rogue wave profiles. Similarly, in Fig. 2(b), $a+b=5$, and keeping $\kappa_{2}=0.6$, the first frequency component becomes $\kappa_{1}=0.9$. Now, of the five first-order components, three of them are merged, forming a second-order profile. The remaining two are symmetrically located on each side of the $x=0$ line. The same rule applies to Fig. 2(c). Here, the frequencies $\kappa_{1}$ and $\kappa_{2}$ also remain within the MI band.

As an illustration of merging of the three first-order rogue components into a single second-order rogue profile we take $\kappa_{1} \rightarrow 0$ in Eq. (9). Applying this limit reduces the two-breather solution, Eq. (9), into a semirational expression that is given by

$$
\begin{aligned}
G_{2}= & -\kappa_{2}^{2}\left[8 \cos \left(t_{\mathrm{s} 2} \kappa_{2}\right) \delta_{2}\right. \\
& \left.+\cosh \left(V_{H 2} x_{\mathrm{s} 2}\right) \kappa_{2}\left(D_{1} \kappa_{2}^{2}-8\right)\right], \\
H_{2}= & -2 \kappa_{2}^{2}\left\{\sinh \left(V_{H 2} x_{\mathrm{s} 2}\right) D_{1} \delta_{2} \kappa_{2}\right. \\
& \left.+16 B x_{\mathrm{s} 1}\left[\cos \left(t_{\mathrm{s} 2} \kappa_{2}\right) \delta_{2}-\cosh \left(V_{H 2} x_{\mathrm{s} 2}\right) \kappa_{2}\right]\right\}, \\
D_{2}= & 2\left\{\cos \left(t_{\mathrm{s} 2} \kappa_{2}\right) \delta_{2}\left(D_{1} \kappa_{2}^{2}-16\right)\right. \\
& +\kappa_{2}\left[-16\left(\sin \left(t_{\mathrm{s} 2} \kappa_{2}\right) t_{\mathrm{s} 1}+2 B \sinh \left(V_{H 2} x_{\mathrm{s} 2}\right) x_{\mathrm{s} 1}\right) \delta_{2}\right. \\
& \left.\left.+\cosh \left(V_{H 2} x_{\mathrm{s} 2}\right)\left(16+\left(D_{1}-4\right) \kappa_{2}^{2}\right)\right]\right\} .
\end{aligned}
$$

Note that the denominator $D_{1}$ from Eq. (8) appears repeatedly in Eq. (11). The limit $\kappa_{1} \rightarrow 0$ transforms one of the breather components of Eq. (9) into a rogue wave profile with a time delay $x_{2}=5$ which is clearly shown in Fig. 3(a). Now, when choosing zero time delay $x_{2}=0$, the three central first-order rogue wave components merge into a second-order profile shown in Fig. 3(b). When $\alpha_{4}=0$, both expressions, Eq. (9) and Eq. (8), reduce to the expression for the standard NLSE [30].

\section{A. Solitons in the limits of breathers}

We have seen that in the first-order breather solution, Eq. (2), the argument $V_{H}$ in the hyperbolic function plays a special role in transforming breather into periodic solitonic structures by unlocking the temporal localization in $x$. Similarly, the argument $V_{H 1}$ in the hyperbolic function in the second-order solution of Eq. (9) influences the breathers and their dynamics. The field distribution of the second-order breather solution becomes increasingly complex.

The second-order breather-to-soliton transformation can be seen when comparing Figs. 2 and 4. For example, comparison of Figs. 2(a) and 4(a) clearly shows solitonlike tails attached to the central peaks. The second-order profile in each period in Fig. 4(a) takes the form of a soliton collision structure on a constant background. Two local maxima at each side of the $x=0$ line in Fig. 2(a) become elongated as a result of transformation into solitons, with the central maximum

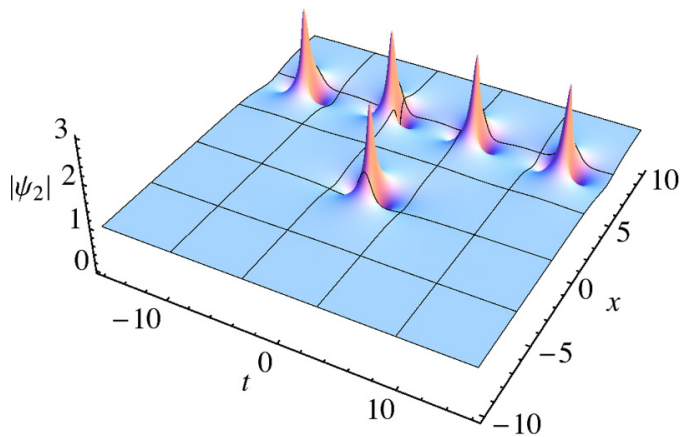

(a)

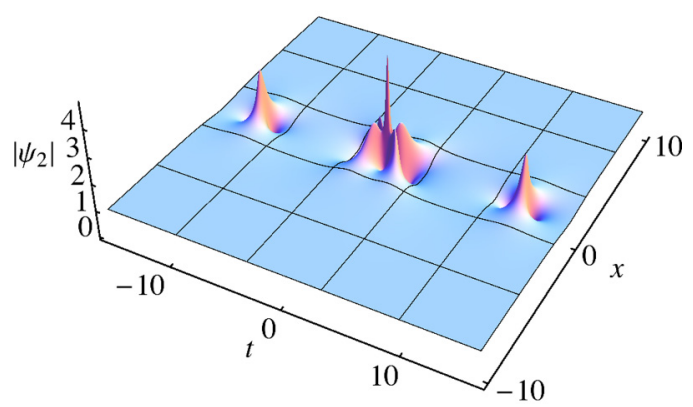

(b)

FIG. 3. Limiting case of the two-breather solution, (9), when $\kappa_{1} \rightarrow 0, \kappa_{2}=0.75, \alpha_{2}=1 / 2$, and $\alpha_{4}=1 / 16$, with (a) $x_{2}=5$ and (b) $x_{2}=0$.

remaining intact. This happens because we keep the same frequency ratio in Figs. 2(a) and 4(a).

Mathematically, the transformation occurs due to the argument $V_{H 1}=0$ in Eq. (9) as well as the parametric relation $\alpha_{2}=\alpha_{4}\left(\kappa_{1}^{2}-6\right)$. Therefore, the two-breather solution takes the form

$$
\begin{aligned}
G_{2}= & \frac{1}{\kappa_{1} \kappa_{2}}\left(\kappa_{1}^{2}-\kappa_{2}^{2}\right)\left\{-\delta_{2} \kappa_{1}^{3} \cos \left(t_{\mathrm{s} 2} \kappa_{2}\right)\right. \\
& \left.+\kappa_{2} \cosh \left(V_{H 3} x_{s 2}\right)\left[\kappa_{1}^{3}+\left(\cos \left(t_{\mathrm{s} 1} \kappa_{1}\right) \delta_{1}-\kappa_{1}\right) \kappa_{2}^{2}\right]\right\} \\
H_{2}= & \frac{\delta_{2}\left(\kappa_{1}^{2}-\kappa_{2}^{2}\right) 2 \sinh \left(V_{H 3} x_{s 2}\right)\left[\kappa_{1}-\cos \left(t_{\mathrm{s} 1} \kappa_{1}\right) \delta_{1}\right]}{\kappa_{1}} \\
D_{2}= & \frac{1}{\kappa_{1} \kappa_{2}}\left\{4 \delta_{1} \delta_{2} \kappa_{1} \kappa_{2} \sin \left(t_{\mathrm{s} 1} \kappa_{1}\right) \sin \left(t_{\mathrm{s} 2} \kappa_{2}\right)\right. \\
& +\kappa_{2}\left[\kappa_{1}^{3}\left(\kappa_{2}^{2}-2\right) \cosh \left(V_{H 3} x_{s 2}\right)-2 \kappa_{1} \kappa_{2}^{2}\right. \\
& \left.+2 \delta_{1}\left(\kappa_{2}^{2}-\kappa_{1}^{2}\right) \cos \left(t_{\mathrm{s} 1} \kappa_{1}\right)\right]+2 \delta_{2} \cos \left(t_{\mathrm{s} 2} \kappa_{2}\right) \\
& \left.\times\left[\kappa_{1}^{3}-\kappa_{1} \kappa_{2}^{2}+\delta_{1}\left(\kappa_{1}^{2}+\kappa_{2}^{2}\right) \cos \left(t_{\mathrm{s} 1} \kappa_{1}\right)\right]\right\}
\end{aligned}
$$

Taking into account the parametric relation $\alpha_{2}=\alpha_{4}\left(\kappa_{1}^{2}-6\right)$, the argument $V_{H_{2}}$ in the hyperbolic function in Eq. (9) takes the new form $V_{H 3}=2 \delta_{2} \alpha_{4}\left(\kappa_{2}^{2}-\kappa_{1}^{2}\right)$.

Comparing Figs. 2(b) and 4(b), where the frequency ratio $\kappa_{1}: \kappa_{2}=3: 2$, we can see that all second-order rogue wave profiles are transformed into a periodic train of soliton collisions, while the two first-order rogue wave profiles between them merge to form parallel low-amplitude solitons.

Similar transformations are shown in Fig. 2(c), where $\kappa_{1}: \kappa_{2}=5: 3$. The second-order rogue wave profiles are 


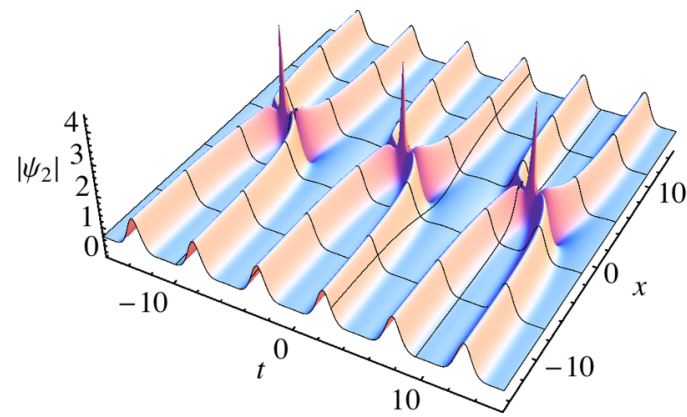

(a)

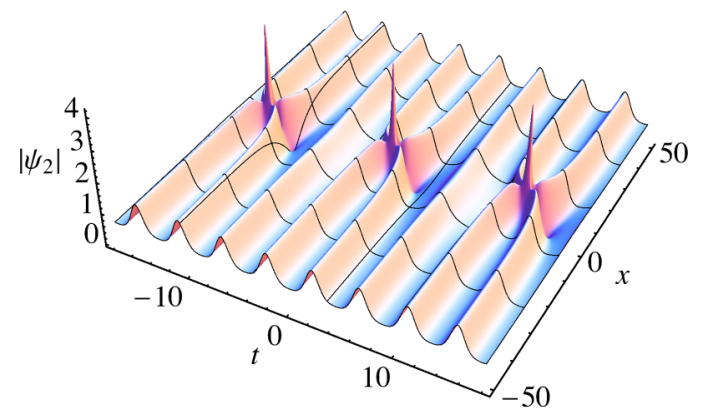

(b)

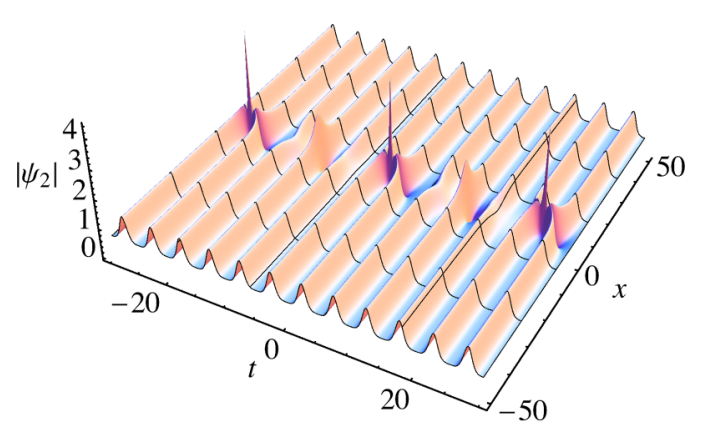

(c)

FIG. 4. Two-breather solution, (12), for the same set of integer modulation frequency ratios as in Fig. 2, namely, (a) $\kappa_{1}=1.2, \kappa_{2}=$ 0.6 , and $\alpha_{4}=1 / 16$; (b) $\kappa_{1}=1.425, \kappa_{2}=0.95$, and $\alpha_{4}=1 / 16$; and (c) $\kappa_{1}=1.25, \kappa_{2}=0.75$, and $\alpha_{4}=1 / 4$.

transformed into soliton collisions as we can see in Fig. 4(c). The nearby paired first-order rogue wave profiles become parallel solitons. The middle one maintains a rogue wave structure and expands at each side of the $x=0$ line as solitons. Two of these profiles are shown in Fig. 4(c).

Parameters $x_{j}$ and $t_{j}$ specify the position of the $j$ th breather in the $(x, t)$ plane. A difference in the positions may cause corresponding shifts. For example, in Fig. 1(a), it introduces a delay of the second breather, with $x_{2}=-5$, relative to the first one. When the two breathers overlap, the delay causes deformation of the wave profile. This case is shown in Fig. 5(a), where the shift $t_{1}=-5$ deformed the solution, which otherwise would have the profile shown in Fig. 4(a). The phase difference between the two breathers causes the high-amplitude features in Fig. 4(a) to disappear. A similar transformation due to the delay is shown in Fig. 5(b). Comparison with Fig. 4(b) shows that the constructive interference here disappears.

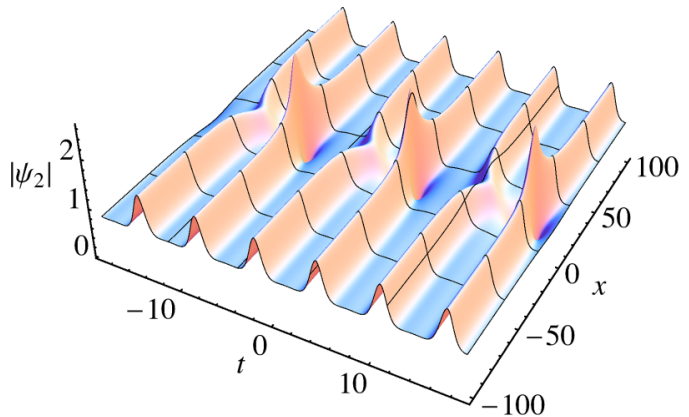

(a)

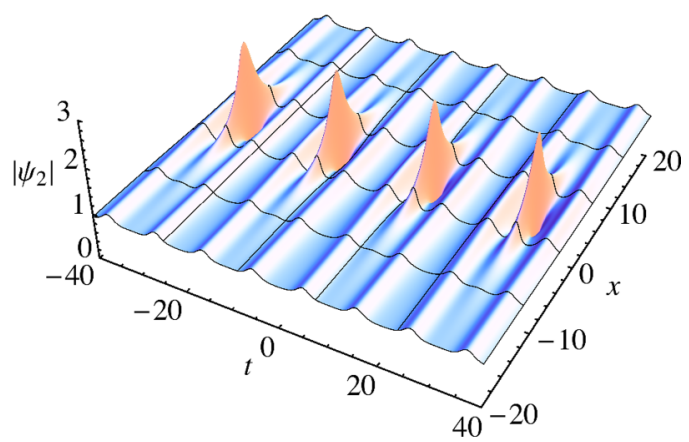

(b)

FIG. 5. Two-breather solution, (12), with the transverse shift $t_{1}=-5$. Other parameters are (a) $\kappa_{1}=0.6, \kappa_{2}=0.9, \alpha_{4}=1 / 16$ and (b) $\kappa_{1}=1.0, \kappa_{2}=0.50, \alpha_{4}=1 / 8$.

Each of the two breathers in the superposition can be transformed into a KM soliton. The periodicity and the localization of an Akhmediev breather and a KM soliton are opposite to each other. While an $\mathrm{AB}$ is periodic in $t$ and localized in $x$, a KM soliton is periodic in $x$ and localized in $t$ [7]. On the other hand, when $\alpha_{2}=\alpha_{4}\left(\kappa_{1}^{2}-6\right)$ in Eq. (12), the first breather component becomes a periodic train of solitons. When, additionally, $\kappa_{2}=i \kappa_{2}$, the second breather related to $\kappa_{2}$ becomes a KM soliton. This case, shown in Fig. 6, corresponds to the interaction between a periodic soliton train and a KM soliton.

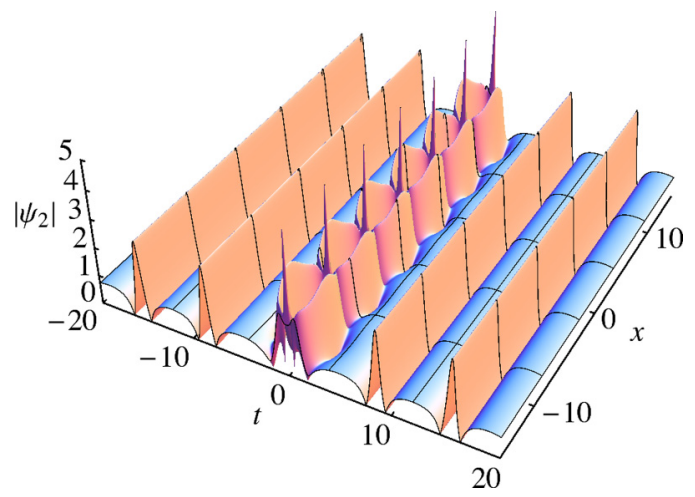

FIG. 6. Interaction between a periodic train of solitons with $\kappa_{1}=0.9$ and a $\mathrm{KM}$ soliton with $\kappa_{2}=1.1 i$ given by Eq. (12). Parameter $\alpha_{4}=1 / 8$. 


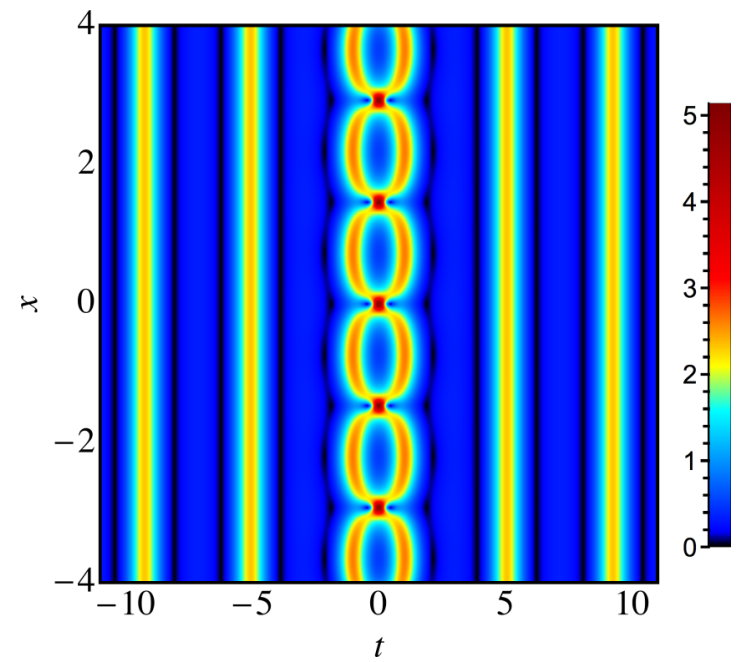

FIG. 7. Plot of the same structure as in Fig. 6, but with $\kappa_{1}=1.5$, $\kappa_{2}=1.98 i$, and $\alpha_{4}=1 / 8$.

In Fig. 6, the transformed solitons are periodic in the $t$ direction, while they maintain their solitonic profile along $x$. This becomes clear if we compare Fig. 1(b), where an AB is interacting with a KM soliton, with Fig. 6. The AB in Fig. 1(b) is transformed into the periodic train of solitons in Fig. 6 following Eq. (12). The complex profile around $t=0$ in Fig. 6 is the result of strong interaction between the KM soliton and the train of solitons. The KM soliton induces periodicity in the combined solution and creates a pulsating profile along the $x$ axis. Figure 7 confirms the features of the complex structure shown in Fig. 6.

Now, taking $\kappa_{1} \rightarrow 0$ in Eq. (12), we obtain

$$
\begin{aligned}
G_{2}= & -\kappa_{2}^{2}\left[8 \cos \left(t_{\mathrm{s} 2} \kappa_{2}\right) \delta_{2}+\cosh \left(V_{H 4} x_{\mathrm{s} 2}\right) \kappa_{2}\left(8-\kappa_{2}^{2} D_{1}^{\prime}\right)\right], \\
H_{2}= & 2 \delta_{2} \kappa_{2}^{3} D_{1}^{\prime} \sinh \left(V_{H 4} x_{\mathrm{s} 2}\right), \\
D_{2}= & 2 \cosh \left(V_{H 4} x_{\mathrm{s} 2}\right) \kappa_{2}\left[16+\kappa_{2}^{2}\left(D_{1}^{\prime}-4\right)\right] \\
& +2 \delta_{2}\left[-16 \sin \left(t_{\mathrm{s} 2} \kappa_{2}\right) t_{\mathrm{s} 1} \kappa_{2}+\cos \left(t_{\mathrm{s} 2} \kappa_{2}\right)\left(\kappa_{2}^{2} D_{1}^{\prime}-16\right)\right],
\end{aligned}
$$

where $V_{H 4}=2 \alpha_{4} \delta_{2} \kappa_{2}^{2}$. In the limit $\kappa_{1} \rightarrow 0$, the relation $\alpha_{2}=\alpha_{4}\left(\kappa_{1}^{2}-6\right)$ in Eq. (12) reduces to $\alpha_{2}=-6 \alpha_{4}$ in Eq. (13) and $D_{1} \rightarrow D_{1}^{\prime}$, where $D_{1}^{\prime}=1+4 t_{s 1}^{2}$. This solution is shown in Fig. 8(a). Here, the period of the soliton train goes to infinity, and only one soliton localized at $t=0$ remains. It interacts with a single $\mathrm{AB}$ located at $x=0$. There is a high-amplitude peak at the point of intersection. Parameter $\kappa_{2}$ controls both the period of the $\mathrm{AB}$ and the amplitude of the soliton. When $\kappa_{2}$ is reduced, the amplitude of the soliton also goes down as shown in Fig. 8(b). On the contrary, when $\kappa_{2}$ is increased, the soliton amplitude also increases as shown in Fig. 8(c).

Solution (13) can also be derived from Eq. (11) by taking the stretching factor $B=0$ due to the relation $\alpha_{2}=-6 \alpha_{4}$. Transforming the isolated first-order rogue wave in Fig. 3(a) into a soliton will provide the same result as in Fig. 8(a). Translations $t_{j}$ and $x_{j}$ have similar effects on this solution as described in connection with Figs. 5(a) and 5(b). Namely,

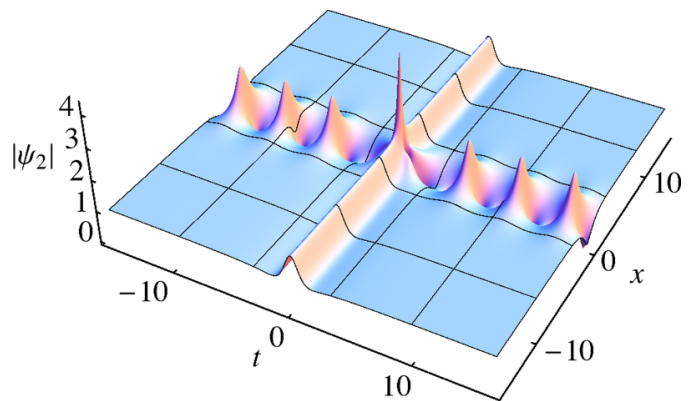

(a)

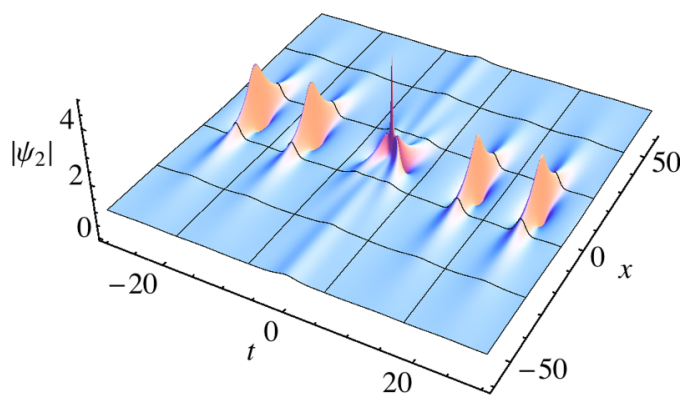

(b)

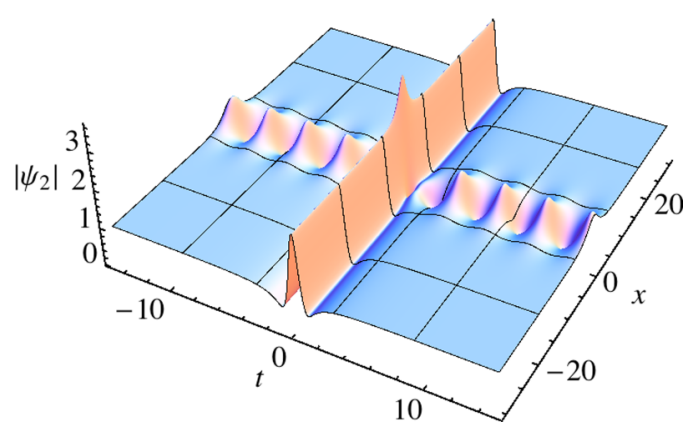

(c)

FIG. 8. Two-breather solution, (13), in the limit $\kappa_{1} \rightarrow 0$. The second breather frequency (a) $\kappa_{2}=1.5$, (b) $\kappa_{2}=0.6$, and (c) $\kappa_{2}=$ 1.9. The equation parameter $\alpha_{4}=1 / 8$.

the $t$ shift applied to the soliton in Fig. 8(a) changes the profile periodically. However, the $x$ shift applied to the breather does not change the profile of the collision point between the soliton and the AB. The same considerations are applicable to Figs. 8(b) and 8(c).

There is one more aspect of solution (13) worthy of mention. Changing $\kappa_{2} \rightarrow i \kappa_{2}$ transforms the AB to a KM soliton. Figure 9 shows the result. The solution now consists of two types of solitons. One is periodic while the other has a fixed profile. This is obvious when the two solitons are well separated as in Fig. 9(a). However, the result is a simply periodic solution when the two solitons are located at the same position in $t$ as in Fig. 9(b). Pulsations here are significantly more complicated than for a single KM soliton, although the period stays the same. Localization in $t$ for this solution is controlled by $\kappa_{2}$. In Fig. $10, \kappa_{2}=1.98 i$, compressing the transverse dimension to the interval $[-2,2]$. In Fig. 11, where the lower value of $\kappa_{2}=0.65 i$ is chosen, the localization is within the larger interval $[-5,5]$. 


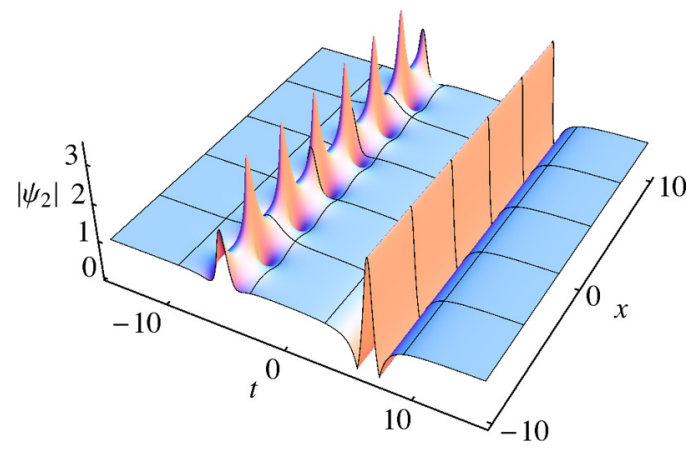

(a)

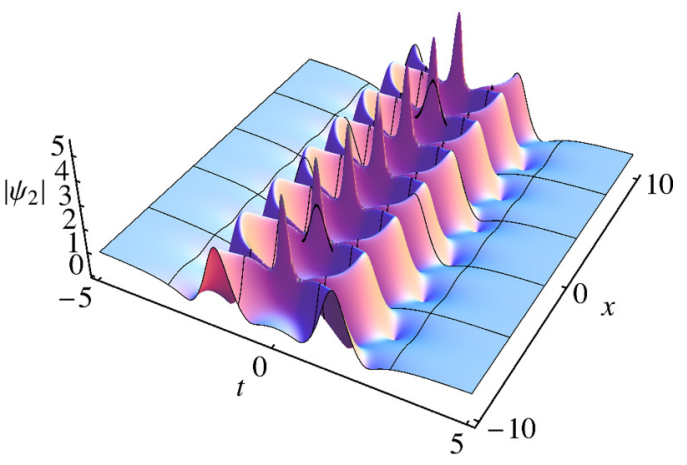

(b)

FIG. 9. Two-breather solution, (13), in the limit $\kappa_{1} \rightarrow 0$ when $\alpha_{4}=1 / 4$. Other parameters are (a) $\kappa_{2}=1.5 i, t_{1}=5, t_{2}=-5$ and (b) $\kappa_{2}=1.5 i, t_{1}=0, t_{2}=0$.

\section{B. Degenerate two-breather solution}

The general breather solution, Eq. (9), is controlled by two independent frequencies $\kappa_{1}$ and $\kappa_{2}$. However, there is one subtlety. When $\kappa_{1} \rightleftharpoons \kappa_{2}$, the solution is undefined. Nevertheless, using l'Hôspital's rule, the solution can be recovered even in this case. In order to do this, we put $\kappa_{1}=\kappa$ and $\kappa_{2}=\kappa+\epsilon$ with small $\epsilon$ in Eq. (9) and carry out Taylor

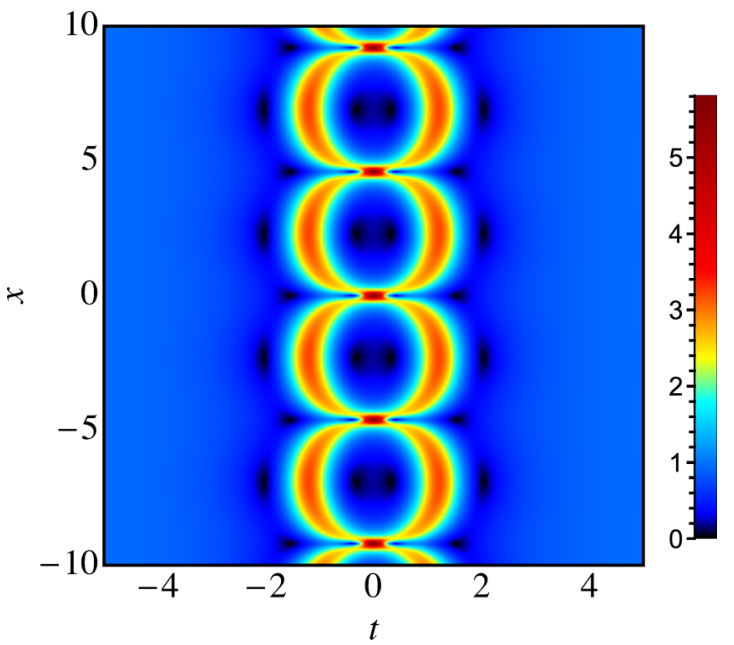

FIG. 10. Pulsating solution, (13), created by merging a KM and a fixed-shape soliton on a constant background. Here the modulation frequency $\kappa_{2}=1.98 i$, while $\alpha_{4}=1 / 16$.

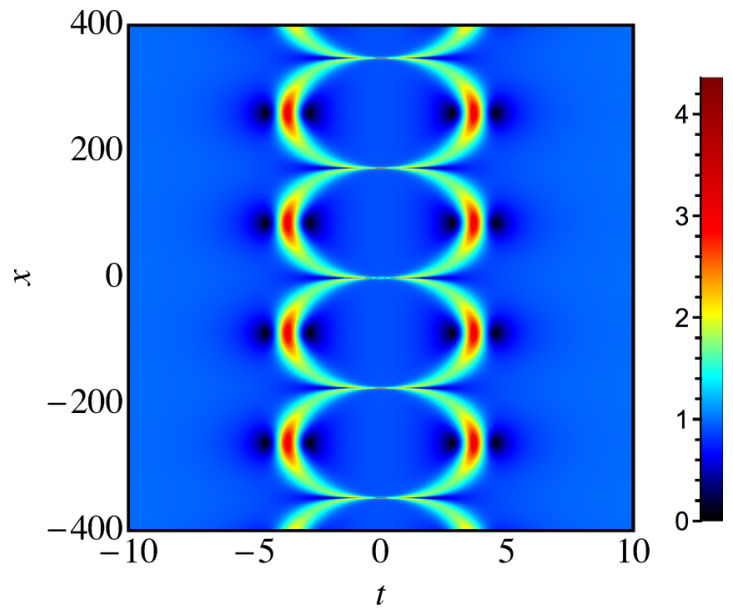

FIG. 11. Same plot as in Fig. 10, except $\kappa_{2}=0.65 i$.

expansion, where we keep only the lowest-order term in $\epsilon$. Following these steps gives us the expressions

$$
\begin{aligned}
G_{2}= & \frac{1}{2 \delta}\left[-\kappa \cosh \left(x V_{H}\right)\left[\left(8 \delta^{2}+\kappa^{4}\right) \cos (t \kappa)\right.\right. \\
& \left.+4 \delta \kappa\left(-2 \cosh \left(x V_{H}\right)+t \delta \sin (t \kappa)\right)\right] \\
& \left.+\kappa \Omega \cos (t \kappa) \sinh \left(x V_{H}\right)\right], \\
H_{2}= & \frac{1}{\delta \kappa}\left[\Omega\left[\delta \cos (t \kappa) \cosh \left(x V_{H}\right)-\kappa\right]\right. \\
& -4 \delta^{3}(\cos (t \kappa)+t \kappa \sin (t \kappa)) \sinh \left(x V_{H}\right) \\
& \left.-\frac{1}{2} \kappa\left(-4 \delta^{2}+\kappa^{4}\right) \sinh \left(2 x V_{H}\right)\right], \\
D_{2}= & \frac{1}{8 \delta^{2} \kappa^{2}}\left\{-x^{2}\left(-4 \delta^{2}+\kappa^{4}\right)^{2} V_{H}^{2}\right. \\
& +2\left[-\kappa^{6}+2 \delta^{2} \kappa^{2}\left(-6+\kappa^{2}\right)+\delta^{4}\left(12-8 t^{2} \kappa^{2}\right)\right. \\
& +4 \delta^{4} \cos (2 t \kappa)+\left[8 \delta^{4}-\kappa^{6}+2 \delta^{2} \kappa^{2}\left(-6+\kappa^{2}\right)\right] \\
& \times \cosh \left(2 x V_{H}\right)+2 \delta \kappa \cosh \left(x V_{H}\right) \\
& \times\left[\left(12 \delta^{2}-12 \kappa^{2}+5 \kappa^{4}\right) \cos (t \kappa)+8 t \delta^{2} \kappa \sin (t \kappa)\right] \\
& -4 \delta \kappa \Omega \cos (t \kappa) \sinh \left(x V_{H}\right) \\
& \left.\left.+16 x \delta^{3} \kappa^{2} \alpha_{4}\left(-\Omega+8 x \delta^{3} \kappa^{2} \alpha_{4}\right)\right]\right\},
\end{aligned}
$$

where $\delta=\frac{\kappa}{2} \sqrt{4-\kappa^{2}}, V_{H}=2 \delta\left[\alpha_{2}-\alpha_{4}\left(\kappa^{2}-6\right)\right]$, and $\Omega=$ $x\left[\left(-4 \delta^{2}+\kappa^{4}\right) V_{H}+16 \delta^{3} \kappa^{2} \alpha_{4}\right]$. Here, the translation parameters $x_{j}=0$ and $t_{j}=0$. The solution contains combinations of trigonometric and hyperbolic functions with only one frequency component $\kappa$. The growth rate for both interacting breathers is the same and given by $\delta$. The contour plot of this solution is shown in Fig. 12(b). Two ABs in this figure, with the same frequency $\kappa$ and with the same growth rate $\delta$, merge at $\psi_{2}(0,0)$ but diverge when $|t|$ is increasing.

Solution (14) is transformed into colliding KM solitons when $\kappa \rightarrow i \kappa$. The growth rate is transformed into the frequency $\delta \rightarrow i \delta$ of the periodic structure along the $x$ axis. This solution is illustrated in Fig. 12(b). When $\alpha_{4}=0$, solution (14) is reduced to the form given in Refs. [30,37]. 


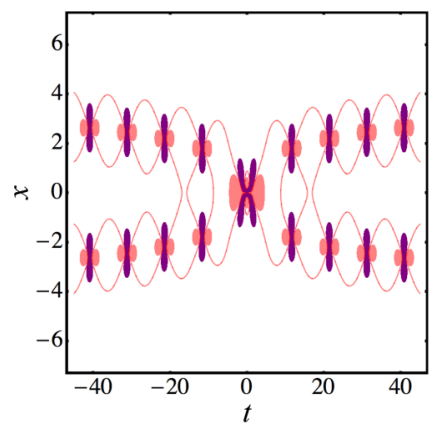

(a)

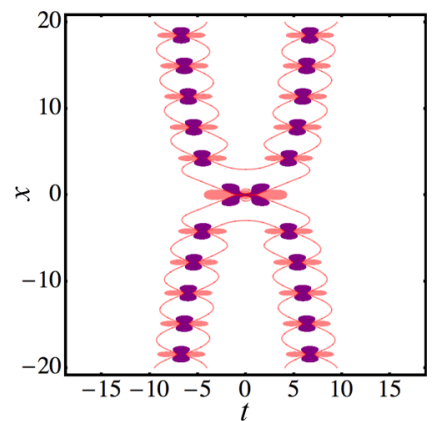

(b)
FIG. 12. Degenerate two-breather solution, (14), with (a) $\kappa=0.65$ and (b) $\kappa=0.65 i$ while translations $t_{1}=t_{2}=x_{1}=x_{2}=0$. In each case, $\alpha_{2}=1 / 2, \alpha_{4}=1 / 8$.

The limit of $\kappa \rightarrow 0$ further reduces solution (14) to the second-order rogue wave:

$$
\begin{aligned}
G_{2}= & -12\left\{-3+8\left[2 t^{4}+t^{2}\left(3+48 B^{2} x^{2}\right)\right.\right. \\
& \left.\left.+4 B x^{2}\left(40 B^{3} x^{2}+9 \alpha_{2}+102 \alpha_{4}\right)\right]\right\} \\
H_{2}= & -48\left\{256 B^{5} x^{5}+x \alpha_{2}\left[-15-24 t^{2}+16 t^{4}\right.\right. \\
& \left.+32 B^{2}\left(1+4 t^{2}\right) x^{2}\right]+6 x \alpha_{4}\left[-23-56 t^{2}+16 t^{4}\right. \\
& \left.\left.+32 B^{2}\left(5+4 t^{2}\right) x^{2}\right]\right\}, \\
D_{2}= & +64 t^{6}+48 t^{4}\left(1+16 B^{2} x^{2}\right) \\
& +12 t^{2}\left[9+256 B^{4} x^{4}-96 B x^{2}\left(\alpha_{2}+22 \alpha_{4}\right)\right] \\
& +16 x^{2}\left[256 B^{6} x^{4}+48 B^{3} x^{2}\left(9 \alpha_{2}+86 \alpha_{4}\right)\right. \\
& \left.+9\left(11 \alpha_{2}^{2}+228 \alpha_{2} \alpha_{4}+1228 \alpha_{4}^{2}\right)\right] .
\end{aligned}
$$

It is shown in Fig. 13. This solution was presented in Refs. [20,23]. It is similar to the second-order AkhmedievPeregrine solution of the NLSE [39,40] except for the stretching factor $B=\alpha_{2}+6 \alpha_{4}$. The role of the stretching factor in generating rogue wave solutions for higher-order equations of the NLSE hierarchy has been discussed in Refs. [24,25]. Briefly, all even-order terms add stretching to the solution profile, while odd-order terms add velocity. When $\alpha_{4}$ is 0 , Eq. (15) becomes the second-order Akhmediev-Peregrine solution of the standard NLSE $[13,41]$. It is assumed here that $\alpha_{2}=1 / 2$ [10].

Now returning to Eq. (14), clearly it contains trigonometric and hyperbolic functions. The argument of the trigonometric functions depends on the variable $t$, and therefore the solution

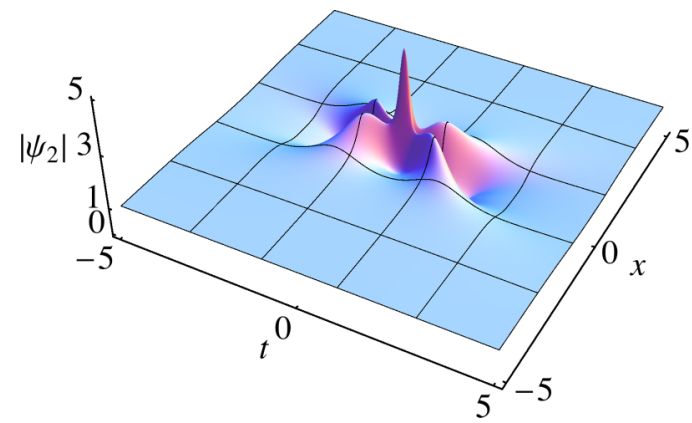

FIG. 13. Second-order rogue-wave solution of Eq. (1) with $\alpha_{2}=$ $1 / 2$ and $\alpha_{4}=1 / 16$ given by Eq. (15).

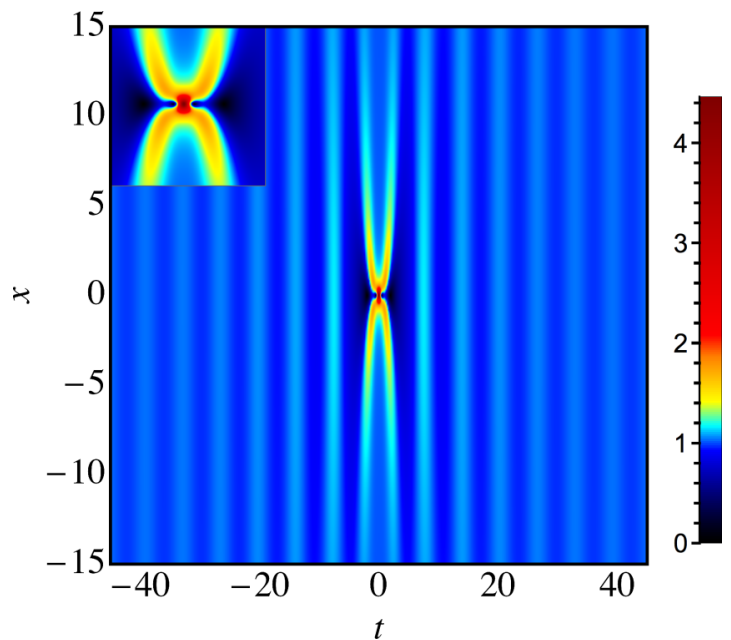

FIG. 14. Plot of the $t$-periodic solution, (16), with frequency $\kappa=1.0$ and $\alpha_{4}=1 / 8$.

is periodic along the $t$ axis. The argument of the hyperbolic functions contains the $x$ variable with coefficient $V_{H}$. Thus, the solution is localized in $x$. The trick is that the expression for $V_{H}$ depends on both $\alpha_{2}$ and $\alpha_{4}$. It may become 0 when $\alpha_{2}=\alpha_{4}\left(\kappa^{2}-6\right)$. Then Eq. (14) reduces to

$$
\begin{aligned}
G_{2}= & -\frac{1}{2 \delta} \kappa\left\{\left(8 \delta^{2}+\kappa^{4}\right) \cos (t \kappa)+4 \delta \kappa[-2+t \delta \sin (t \kappa)]\right\}, \\
H_{2}= & 16 x \delta^{2} \kappa[-\kappa+\delta \cos (t \kappa)] \alpha_{4}, \\
D_{2}= & \frac{1}{2 \delta^{2} \kappa^{2}}\left\{-\kappa^{6}+2 \delta^{2} \kappa^{2}\left(-6+\kappa^{2}\right)+\delta^{4}\left(10-4 t^{2} \kappa^{2}\right)\right. \\
& +\delta \kappa\left(12 \delta^{2}-12 \kappa^{2}+5 \kappa^{4}\right) \cos (t \kappa)+2 \delta^{3}[\delta \cos (2 t \kappa) \\
& \left.\left.+4 t \kappa^{2} \sin (t \kappa)\right]-64 x^{2} \delta^{6} \kappa^{4} \alpha_{4}^{2}\right\} .
\end{aligned}
$$

This solution is shown in Fig. 14. Comparing Fig. 14 with Fig. 12(a), we note that the localization along the $x$ axis is lost. The two parallel breathers in Fig. 12(a) are transformed into a plane wave in Fig. 14, with low-amplitude ripples on the background. The main feature of this solution is the highamplitude central peak at the origin $(0,0)$. Now, due to the absence of localization along $x$, it looks more like a collision of two solitons as shown in the inset in Fig. 14.

Solution (16) for the higher value of $\kappa=1.7$ is shown in Fig. 15. This profile is similar to the one shown in Fig. 14

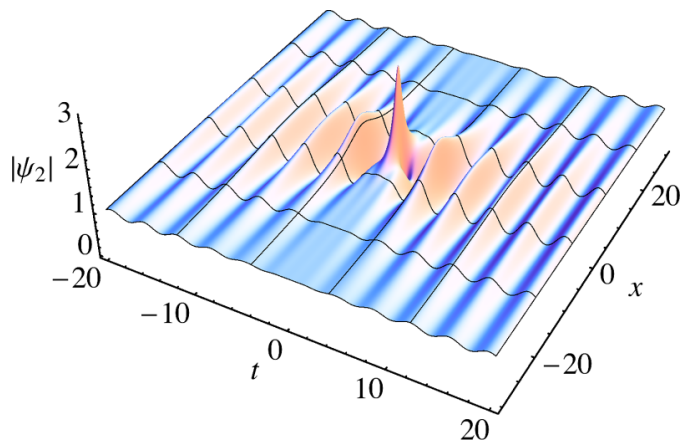

FIG. 15. Two-breather solution, (16), with the higher modulation frequency $\kappa=1.7$ and $\alpha_{4}=1 / 8$. 


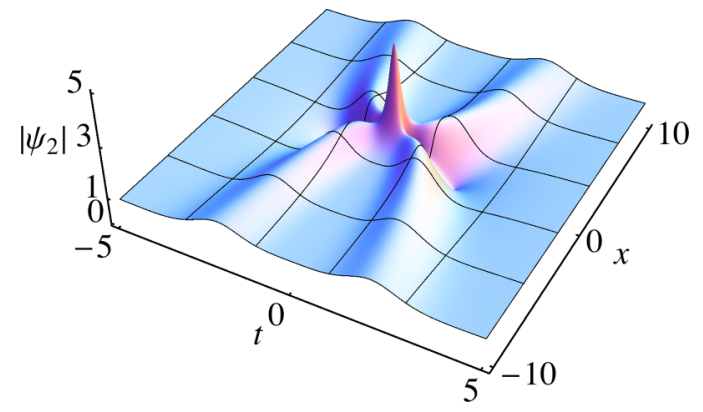

FIG. 16. Solution (17) with $\alpha_{2}=1 / 2$ and $\alpha_{4}=1 / 8$.

except for the weaker periodic modulation of the background and lower amplitude of the central peak. In the limit $\kappa \rightarrow 2$ in Eq. (16), any modulation disappears and only flat background is left.

Another limiting case appears when $\kappa \rightarrow 0$ in Eq. (16). It is given by

$$
\begin{aligned}
& G_{2}=-12\left(-3+24 t^{2}+16 t^{4}\right), \\
& H_{2}=2304\left(1+4 t^{2}\right) x \alpha_{4}, \\
& D_{2}=9+108 t^{2}+48 t^{4}+64 t^{6}+36864 x^{2} \alpha_{4}^{2} .
\end{aligned}
$$

Equation (17) can also be derived from the second-order rogue solution, (15), by taking the stretching factor $B=0$, using the relation $\alpha_{2}=-6 \alpha_{4}$. This solution is shown in Fig. 16. It is reminiscent of the degenerate case of two-soliton collision [42] but occurs on a finite background. The solution, Eq. (17), was obtained recently in Ref. [33].

\section{Influence of differential shifts}

The Taylor expansion that we carried out in deriving Eq. (14) does not allow us to obtain the solution with nonzero translation parameters $x_{j}$ and $t_{j}$. To take their influence into account we have to use the concept of differential shifts $[38,43]$. The latter are modulation-frequency-dependent translations. Applying the concept of differential shift to the degenerate solution requires special treatment [30], as general procedures fail.

Namely, taking series expansion of the numerator comprising $G_{2}$ and $H_{2}$, the lowest-order coefficient remains with $\epsilon^{1}$. Carrying out a similar series expansion on the denominator $D_{2}$, the lowest-order coefficient remains with the term $\epsilon^{0}$ and is $4 \delta^{2}\left\{\cos \left[\kappa\left(t_{1}-t_{2}\right)\right]-\cosh \left[V_{H}\left(x_{1}-x_{2}\right)\right]\right\}$. Applying the l'Hôspital rule when using the Taylor expansion will not produce an appropriate limit unless $x_{1}=x_{2}$ and $t_{1}=t_{2}$ in the denominator. In order to overcome this difficulty, we replace $x_{j}=X_{j} \epsilon$ and $t_{j}=T_{j} \epsilon$ and then perform the series expansion. As a result, we obtain the desired limit of $\left(G_{2}+i H_{2}\right) / D_{2}$ with the lowest-order coefficient at $\epsilon^{1}$ :

$$
\begin{aligned}
G_{2}= & \frac{1}{2 \delta}\left\{\kappa \operatorname { c o s h } ( x V _ { H } ) \left[-\left(8 \delta^{2}+\kappa^{4}\right) \cos (t \kappa)\right.\right. \\
& \left.+8 \delta \kappa \cosh \left(x V_{H}\right)-4 \delta^{2} \kappa\left(t+t_{d} \kappa\right) \sin (t \kappa)\right] \\
& \left.+\kappa \Omega^{\prime} \cos (t \kappa) \sinh \left(x V_{H}\right)\right\}
\end{aligned}
$$

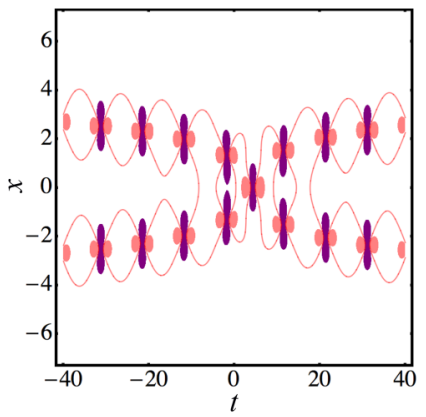

(a)

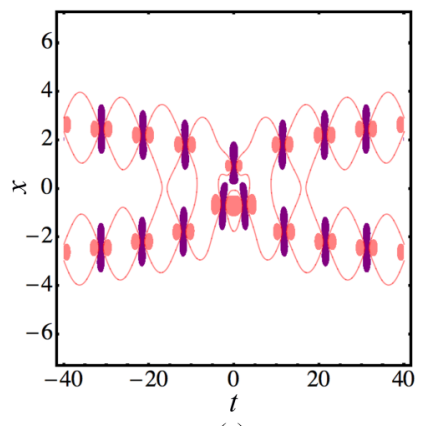

(c)

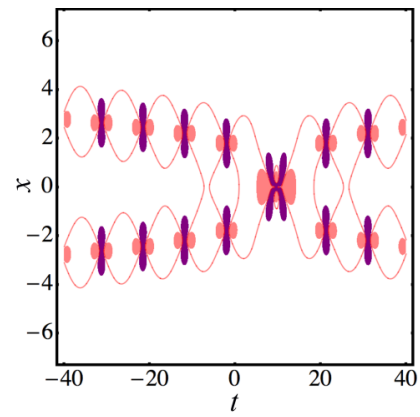

(b)

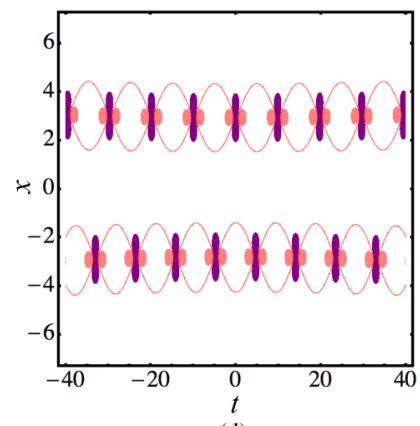

(d)
FIG. 17. Degenerate solution, (18), with nonzero differential shift parameters $t_{d}$ and $x_{d}$ : (a) $t_{d}=-6, x_{d}=0$; (b) $t_{d}=-14.88, x_{d}=0$; (c) $x_{d}=1, t_{d}=0$; and (d) $x_{d}=40, t_{d}=0$. Other parameters are $\kappa=0.65, \alpha_{2}=1 / 2$, and $\alpha_{4}=1 / 8$.

$$
\begin{aligned}
H_{2}= & \frac{1}{\delta \kappa}\left\{\Omega^{\prime}\left[\delta \cos (t \kappa) \cosh \left(x V_{H}\right)-\kappa\right]-4 \delta^{3}[\cos (t \kappa)\right. \\
& \left.+\kappa\left(t+t_{d} \kappa\right) \sin (t \kappa)\right] \sinh \left(x V_{H}\right) \\
& \left.-\frac{1}{2} \kappa\left(-4 \delta^{2}+\kappa^{4}\right) \sinh \left(2 x V_{H}\right)\right\} \\
D_{2}= & \frac{1}{8 \delta^{2} \kappa^{2}}\left\{8 \delta^{4} \cos (2 t \kappa)+2\left[8 \delta^{4}-\kappa^{6}\right.\right. \\
& \left.+2 \delta^{2} \kappa^{2}\left(-6+\kappa^{2}\right)\right] \cosh \left(2 x V_{H}\right) \\
& -8 \delta \Omega^{\prime} \cos (t \kappa) \sinh \left(x V_{H}\right)+4 \delta \kappa \cosh \left(x V_{H}\right) \\
& \times\left[\left(12 \delta^{2}-12 \kappa^{2}+5 \kappa^{4}\right) \cos (t \kappa)\right. \\
& \left.+8 \delta^{2} \kappa \sin (t \kappa)\left(t+\kappa t_{d}\right)\right]-\kappa^{6}\left(2+x^{2} \kappa^{2} V_{H}^{2}\right) \\
& +4 \delta^{2} \kappa^{2}\left[-6+\kappa^{2}+2 x \kappa^{2} V_{H}^{2}\left(x+\kappa x_{d}\right)\right] \\
& -8 \delta^{4}\left[-3+2 t^{2} \kappa^{2}+2 \kappa^{3} t_{d}\left(2 t+\kappa t_{d}\right)\right. \\
& \left.\left.+2 V_{H}^{2}\left(x+\kappa x_{d}\right)^{2}\right]-32 x \delta^{3} \kappa^{2} \alpha_{4}\left(\Omega^{\prime}-8 x \delta^{3} \kappa^{2} \alpha_{4}\right)\right\}
\end{aligned}
$$

where $x_{d}=\left(X_{1}-X_{2}\right), t_{d}=\left(T_{1}-T_{2}\right)$, and $\Omega^{\prime}=x \kappa^{2}\left(\kappa^{2} V_{H}+\right.$ $\left.16 \delta^{3} \alpha_{4}\right)-4 \delta^{2} V_{H}\left(x+\kappa x_{d}\right)$. The parameter $\delta$ remains the same as in Eq. (14). Equations (18) contain additional free parameters, $x_{d}$ and $t_{d}$. When these are 0, Eqs. (18) are the same as Eqs. (14).

How do the values $t_{d}$ and $x_{d}$ affect the solution? Figure 17 provides a few illustrations answering this question. The parameter $t_{d}$ shifts one breather relative to the other. For example, a small shift may unbalance the synchronization 
achieved in Fig. 12(a). Comparing Fig. 17(a) with Fig. 12(a), we can see that for the small value of $t_{d}=-6$ and $x_{d}=0$, the central second-order profile breaks into three first-order rogue waves. As the breathers are periodic, a larger value of $t_{d}$ may restore the synchronization. This is achieved precisely at the value $t_{d}=-14.88$ as shown in Fig. 17(b). The second-order structure is restored here but shifted along the $t$ axis to the right. Disintegration and restoration of the second-order structure occur periodically with a change in the parameter $t_{d}$ value. The use of a negative value of $t_{d}$ results in a move in the positive $t$ direction. A negative sign will reverse the direction of this move.

The differential shift $x_{d}$ separates the two breathers along the $x$ axis. This is shown clearly in Figs. 17(c) and 17(d). With a small separation, when $x_{d}=1$, the central second-order structure splits into three first-order rogue waves. This is shown in Fig. 17(c). When the separation is larger, $x_{d}=40$, the two breathers are completely separated as Fig. 17(d) demonstrates. Changing the sign of $x_{d}$ leads again to separation of the two breathers, with the patterns reversed in $x$. Thus, changing either of the parameters, $t_{d}$ or $x_{d}$, may break apart the second-order profile at the origin. However, the reason for the splitting in each case is different. Unlike changing $t_{d}$, increasing the value of $x_{d}$ cannot restore the triplets to form a second-order profile periodically.

Changing $\kappa \rightarrow i \kappa$ in Eq. (18) transforms $\mathrm{ABs}$ to $\mathrm{KM}$ solitons. This action introduces the $90^{\circ}$ rotation shown in Fig. 12. This is explained in Sec. III B. In order to see the effect of the differential shift parameters on KM solitons we must also change $t_{d} \rightarrow i t_{d}$ and $x_{d} \rightarrow i x_{d}$. When these parameters are varied, the degenerate $\mathrm{KM}$ solution experiences effects analogous to those shown in Fig. 17.

The limit $\kappa \rightarrow 0$ in Eq. (18) is also not simple. In order to approach this limit, the coefficient in front of the differential shift parameter must be $\sim \kappa^{2}$ [43]. In Eq. (18), the coefficients in front of $t_{d}$ and $x_{d}$ are $\sim \kappa$ and taking the limit will lead us to a plane wave solution unless we replace $t_{d} \rightarrow k t_{d}^{\prime}$ and $x_{d} \rightarrow k x_{d}^{\prime}$, thus defining the coefficients in front of $t_{d}^{\prime}$ and $x_{d}^{\prime} \sim \kappa^{2}$. Now taking the limit $\kappa \rightarrow 0$ in Eq. (18) reduces it to a second-order triplet solution:

$$
\begin{aligned}
G_{2}= & -12\left\{-3+8\left[2 t^{4}+160 B^{4} x^{4}+t^{2}\left(3+48 B^{2} x^{2}\right)\right.\right. \\
& \left.\left.-24 t t_{d}+12 B x\left(-8 B x_{d}+3 x \alpha_{2}+34 x \alpha_{4}\right)\right]\right\}, \\
H_{2}= & -48\left\{256 B^{5} x^{5}+24 B\left(1+4 t^{2}-16 B^{2} x^{2}\right) x_{d}\right. \\
& +x\left[-15-24 t^{2}+16 t^{4}+32 B^{2}\left(1+4 t^{2}\right) x^{2}\right. \\
& \left.-192 t t_{d}\right] \alpha_{2}+6 x\left[-23-56 t^{2}+16 t^{4}\right. \\
& \left.\left.+32 B^{2}\left(5+4 t^{2}\right) x^{2}-192 t t_{d}\right] \alpha_{4}\right\}, \\
D_{2}= & +64 t^{6}+4096 B^{6} x^{6}+48 t^{4}\left(1+16 B^{2} x^{2}\right) \\
& +12 t^{2}\left(9+256 B^{4} x^{4}\right) \\
& +48 t_{d}\left\{4 t\left(-3+4 t^{2}-48 B^{2} x^{2}\right)\right. \\
& +48 t_{d}^{2}+192 B^{2} x_{d}^{2}-16 B x x_{d}\left[9 \alpha_{2}\right. \\
& \left.+2\left(-6 B t^{2}+8 B^{3} x^{2}+51 \alpha_{4}\right)\right] \\
& +x^{2}\left[33 \alpha_{2}^{2}+12 \alpha_{2}\left(-2 B t^{2}+12 B^{3} x^{2}+57 \alpha_{4}\right)\right. \\
& \left.\left.+4 \alpha_{4}\left(-132 B t^{2}+344 B^{3} x^{2}+921 \alpha_{4}\right)\right]\right\},
\end{aligned}
$$

where, for simplicity, we have omitted the primes over the free parameters $t_{d}$ and $x_{d}$. This solution is shown in Fig. 18. It was

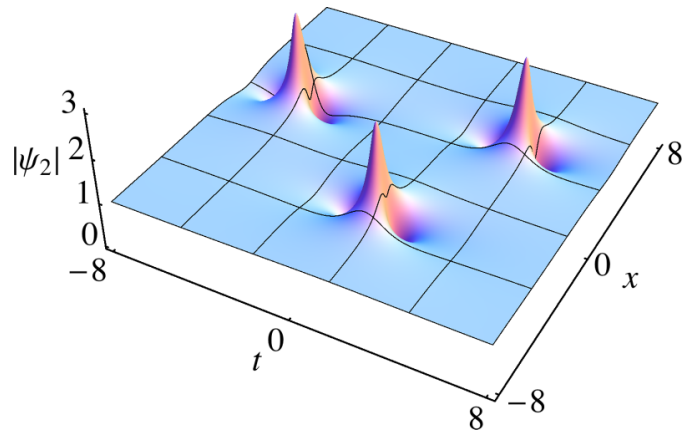

FIG. 18. Rogue-wave triplet, (19), for $x_{d}=25, t_{d}=-25, \alpha_{2}=$ $1 / 2$, and $\alpha_{4}=1 / 32$.

given in Refs. [20,23]. As shown in Fig. 18, the differential shift parameters $x_{d}$ and $t_{d}$ break apart the second-order profile shown in Fig. 13 and disintegrates it into three separate firstorder rogue waves. Naturally, if we take $x_{d}=t_{d}=0$, Eq. (19) will reduce to Eq. (15) and the triplet will merge and form a second-order rogue wave solution. Taking $\alpha_{4}=0$ in Eq. (19) will further reduce the solution to the standard NLSE rogue wave triplet that has been given in Refs. [38,43].

Now, if we look at Eqs. (14) and (18), again, the parameter $V_{H}$ is responsible for localization in $x$. It can be $0, V_{H}=0$, when $\alpha_{2}=\alpha_{4}\left(\kappa^{2}-6\right)$. In this particular case, the solution, Eq. (18), is reduced to

$$
\begin{aligned}
G_{2}= & -\frac{1}{2 \delta} \kappa\left\{\left(8 \delta^{2}+\kappa^{4}\right) \cos (t \kappa)\right. \\
& \left.+4 \delta \kappa\left[-2+\delta \sin (t \kappa)\left(t+\kappa t_{d}\right)\right]\right\}, \\
H_{2}= & 16 x \delta^{2} \kappa[-\kappa+\delta \cos (t \kappa)] \alpha_{4}, \\
D_{2}= & \frac{1}{2 \delta^{2} \kappa^{2}}\left\{-\kappa^{6}+2 \delta^{2} \kappa^{2}\left(-6+\kappa^{2}\right)\right. \\
& +\delta \kappa\left(12 \delta^{2}-12 \kappa^{2}+5 \kappa^{4}\right) \cos (t \kappa) \\
& +2 \delta^{3}\left(\delta \cos (2 t \kappa)+4 \kappa^{2} \sin (t \kappa)\left(t+\kappa t_{d}\right)\right) \\
& \left.-2 \delta^{4}\left[-5+2 \kappa^{2}\left(t+\kappa t_{d}\right)^{2}\right]-64 x^{2} \delta^{6} \kappa^{4} \alpha_{4}^{2}\right\} .
\end{aligned}
$$

Solution (20) is more general than (16), as it has a free parameter $t_{d}$. However, there is no $x_{d}$ in this solution. This

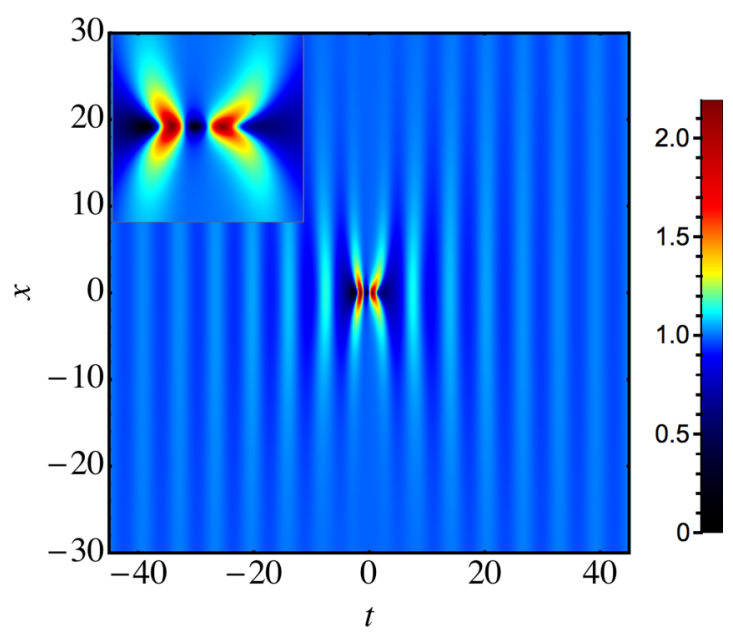

FIG. 19. Plot of degenerate solution, (20), with $t_{d}=0.5$ and $\alpha_{4}=1 / 4$. 


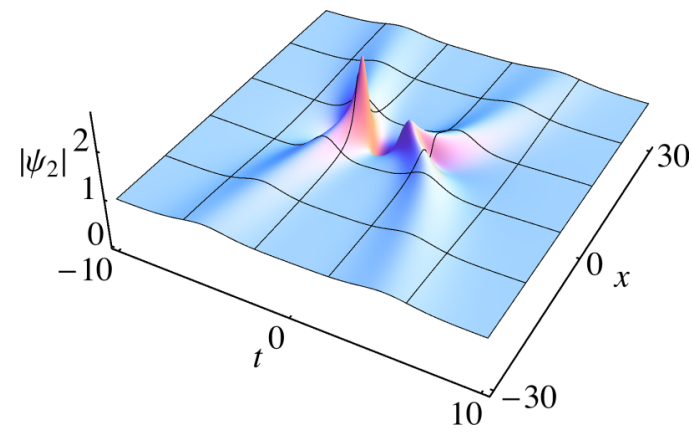

FIG. 20. Transformation of a triplet into a single rogue wave revealed by Eq. (21) with $\alpha_{4}=1 / 4$ when the differential shift parameter is small, $t_{d}=1$.

happens because $x_{d}$ always appears in Eq. (18) in combination with $V_{H}$, which is 0 .

Solution (20) is shown in Fig. 19. It is similar to the one shown in Fig. 14 but the central high-amplitude profile is broken and has shifted along the $t$ axis with the influence of the parameter $t_{d}$. The latter is shown in the inset in Fig. 19. In the limit $\kappa \rightarrow 2$, the modulation amplitude becomes 0 and solution (20) reduces to the background level. In the opposite limit, $\kappa \rightarrow 0$, Eq. (20) reduces to the expressions

$$
\begin{aligned}
G_{2}= & 12\left[3-8 t\left(3 t+2 t^{3}-24 t_{d}\right)\right], \\
H_{2}= & 2304\left(1+4 t^{2}\right) x \alpha_{4}, \\
D_{2}= & 9+108 t^{2}+48 t^{4}+64 t^{6} \\
& +192 t_{d}\left(-3 t+4 t^{3}+12 t_{d}\right)+36864 x^{2} \alpha_{4}^{2} .
\end{aligned}
$$

Similarly to the case of Eq. (18), the limit $\kappa \rightarrow 0$ in Eq. (20) is not simple. We first have to replace $t_{d} \rightarrow k t_{d}^{\prime}$ and only then take the limit $\kappa \rightarrow 0$, thus obtaining Eq. (21). This solution can also be obtained from Eq. (19). In this case, we need to take the stretching factor $B=0$ with the relation $\alpha_{2}=-6 \alpha_{4}$.

The question arises, What happens to the triplet solution in the limit $B \rightarrow 0$ in Eq. (19)? Figure 20 shows clearly that the triplet undergoes a transformation. Two of the first-order rogue waves from the triplet disappear into the background, leaving only one first-order rogue wave. This transformation becomes clearer with a further increase in the value of $t_{d}$ as shown in

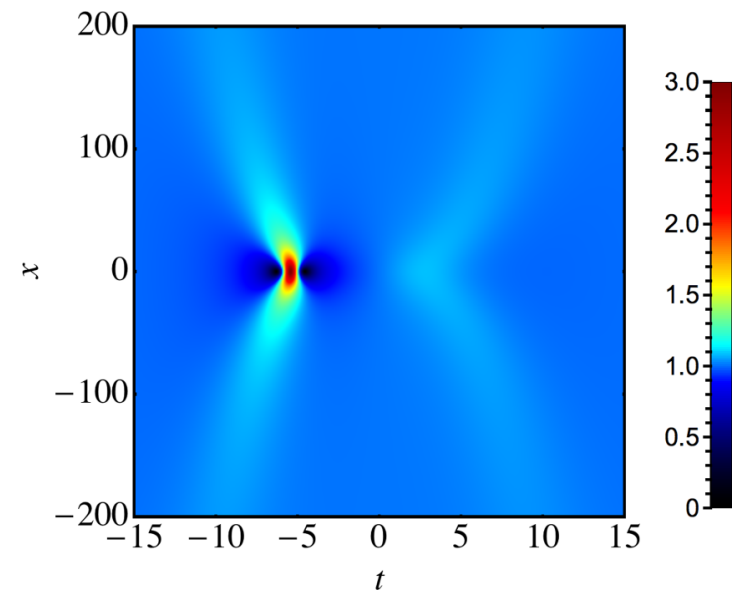

FIG. 21. Plot of solution (21) with a large value of the differential shift parameter, $t_{d}=40$, and $\alpha_{4}=1 / 4$.
Fig. 21. When $t_{d}=40$, only a single rogue wave remains, with the highest amplitude 3, i.e., the same amplitude as for the Peregrine solution. This transformation takes another form when we take $t_{d}=0$, reducing Eq. (21) to Eq. (17).

Thus, the influence of the differential shift parameters $x_{d}$ and $t_{d}$ on the wave profiles is far from trivial. Higherorder solutions $(n>2)$ could be even more complicated. For example, in the case of the NLSE, the $n$ th-order rogue wave solution has $n(n+1) / 2$ peaks due to the influence of the parameters $x_{d}$ and $t_{d}$ [43]. Similar and even more complicated effects can be found for solutions of Eq. (1).

\section{CONCLUSIONS}

Our main results are as follows.

(1) We derive and analyze the first-order breather solution of Eq. (1). Particular cases of this solution are rogue waves and KM solitons. We show that for a specific choice of parameters, the breather solution loses localization but remains periodic. On the other hand, the rogue wave can be transformed to a soliton solution. Moreover, a special choice of equation parameters transforms a periodic KM soliton to a stationary soliton.

(2) We present a general two-breather solution of Eq. (1) which has several independent free parameters. Two of them are the modulation frequencies, which describe the periodicity of each breather evolving on a constant background. Variations of them produce a rich variety of wave profiles.

(3) We derive the degenerate two-breather solution when the modulation frequencies of the two breathers coincide. Several particular cases of this solution are presented. For example, the zero-frequency limit of the degenerate solution leads to a second-order rogue wave solution of Eq. (1).

(4) We introduce two additional differential shift parameters in the degenerate solution and analyze their influence on the wave profile.

These structures and their mutual transformations are relevant to nonlinear optics, plasmas, and hydrodynamics [44] and Bose-Einstein condensates. In optics, these effects can be studied similarly to the higher-order modulation instability that has been found experimentally in Ref. [45]. Turbulence [46-48] and supercontinuum generation [49,50] are two other examples of highly complex nonlinear wave phenomena where our results could be useful.

An important property of the solutions is their stability. There are various approaches to stability problem [51]. One of them is stability relative to small perturbations of the solution [52]. Another one is stability relative to perturbations of the original equation [53]. There is also an approach based on numerical simulations [54]. The problem is far from being simple because the background is involved. Preliminary studies indicate that solitons on a background are stable [55]. However, complex analysis is required which we cannot provide here. Clearly, exact solutions have to be presented first before stability problems can be even posed in accurate form as mathematical problems. We leave these problems for future studies as each of the above mentioned aspects require a separate lengthy investigation which cannot be presented in the frame of a single publication. 


\section{ACKNOWLEDGMENTS}

The authors acknowledge support from the Qatar National Research Fund (Grant No. NPRP 9-020-1-006), Science
Program, Texas A\&M University at Qatar. N.A. acknowledges support from the Australian Research Council (Discovery Project No. DP150102057) and from the Volkswagen Stiftung.
[1] V. I. Bespalov and V. I. Talanov, Filamentary structure of light beams in nonlinear liquids, Pis'ma Zh. Eksp. Teor. Fiz. 3, 471 (1966) [JETP Lett. 3, 307 (1966)].

[2] T. B. Benjamin, J. E. Feir, The disintegration of wave trains on deep water. Part 1. Theory, J. Fluid Mech. 27, 417 (1967).

[3] K. Shimizu and H. Ichikawa, Automodulation of ion oscillation modes in plasma, J. Phys. Soc. Jpn. 33, 789 (1972).

[4] A. Turing, The chemical basis of morphogenesis, Philos. Trans. Roy. Soc. B 237, 37 (1952).

[5] R. M. Miura, H. E. Moses, R. Hermann, A. R. Bishop, H. C. Yuen, B. M. Lake, K. E. Lonngren, H. Ikezi, R. D. Parmentier, D. W. McLaughlin et al., Solitons in Action (Academic Press, New York, 1978).

[6] H. C. Yuen and W. E. Ferguson Jr., Relationship between Benjamin-Feir instability and recurrence in the nonlinear schrödinger equation, Phys. Fluids 21, 1275 (1978).

[7] N. Akhmediev and V. I. Korneev, Modulation instability and periodic solutions of the nonlinear Schrödinger equation, Theor. Math. Phys. 69, 1089 (1986).

[8] N. Akhmediev, V. M. Eleonsky, and N. E. Kulagin, Generation of periodic trains of picosecond pulses in an optical fiber: Exact solutions, Sov. Phys. JETP 62, 894 (1985).

[9] J. M. Dudley, G. Genty, F. Dias, B. Kibler, and N. Akhmediev, Modulation instability, Akhmediev breathers and continuous wave supercontinuum generation, Opt. Express 17, 21497 (2009).

[10] N. Akhmediev and A. Ankiewicz, Solitons: Nonlinear Pulses and Beams (Chapman \& Hall, London, 1997).

[11] E. Fermi, J. Pasta, and S. Ulam, Studies of nonlinear problems. I. Los Alamos Report LA-1940 (1955), published in Collected Papers of Enrico Fermi, edited by E. Segre (University of Chicago Press, Chicago, 1965).

[12] N. Akhmediev, J. M. Soto-Crespo, and A. Ankiewicz, Extreme waves that appear from nowhere: On the nature of rogue waves, Phys. Lett. A 373, 2137 (2009).

[13] N. Akhmediev, A. Ankiewicz, and J. M. Soto-Crespo, Rogue waves and rational solutions of the nonlinear Schrödinger equation, Phys. Rev. E 80, 026601 (2009).

[14] M. J. Potasek and M. Tabor, Exact solutions for an extended nonlinear Schrödinger equation, Phys. Lett. A 154, 449 (1991).

[15] S. B. Cavalcanti, J. C. Cressoni, H. R. da Cruz, and A. S. Gouveia-Neto, Modulation instability in the region of minimum group-velocity dispersion of single-mode optical fibers via an extended nonlinear Schrödinger equation, Phys. Rev. A 43, 6162 (1991).

[16] Y. Kodama, A. V. Mikhailov, and S. Wabnitz, Input pulse optimization in wavelength-division-multiplexed soliton transmissions, Opt. Commun. 143, 53 (1997).

[17] G. P. Agrawal, Nonlinear fiber optics: Its history and recent progress [invited], J. Opt. Soc. Am. B 28, A1 (2011).

[18] G. P. Agrawal, Nonlinear Fiber Optics, 5th ed. (Academic Press, New York, 2012).
[19] K. Porsezian, M. Daniel, and M. Lakshmanan, On the integrability aspects of the one-dimensional classical continuum isotropic biquadratic Heisenberg spin chain, J. Math. Phys. 33, 1807 (1992).

[20] L. H. Wang, K. Porsezian, and J. S. He, Breather and rogue wave solutions of a generalized nonlinear Schrödinger equation, Phys. Rev. E 87, 053202 (2013).

[21] M. Lakshmanan, K. Porsezian, and M. Daniel, Effect of discreteness on the continuum limit of the Heisenberg spin chain, Phys. Lett. A 133, 483 (1988).

[22] A. Ankiewicz and N. Akhmediev, Higher-order integrable evolution equation and its soliton solutions, Phys. Lett. A 378, 358 (2014).

[23] A. Ankiewicz, Y. Wang, S. Wabnitz, and N. Akhmediev, Extended nonlinear Schrödinger equation with higher-order odd and even terms and its rogue wave solutions, Phys. Rev. E 89, 012907 (2014).

[24] D. J. Kedziora, A. Ankiewicz, A. Chowdury, and N. Akhmediev, Integrable equations of the infinite nonlinear Schrödinger equation hierarchy with time variable coefficients, Chaos Interdis. J. Nonlin. Sci. 25, 103114 (2015).

[25] A. Ankiewicz, D. J. Kedziora, A. Chowdury, U. Bandelow, and N. Akhmediev, Infinite hierarchy of nonlinear Schrödinger equations and their solutions, Phys. Rev. E 93, 012206 (2016).

[26] A. Ankiewicz, Soliton, rational, and periodic solutions for the infinite hierarchy of defocusing nonlinear Schrödinger equations, Phys. Rev. E 94, 012205 (2016).

[27] R. Hirota, Exact envelope-soliton solutions of a nonlinear wave equation, J. Math. Phys. 14, 805 (1973).

[28] T. Kano, Normal form of nonlinear Schrödinger equation, J. Phys. Soc. Jpn. 58, 4322 (1989).

[29] L. Wang, J.-H. Zhang, Z.-Q. Wang, C. Liu, M. Li, F.-H. Qi, and Rui Guo, Breather-to-soliton transitions, nonlinear wave interactions, and modulational instability in a higher-order generalized nonlinear Schrödinger equation, Phys. Rev. E 93, 012214 (2016).

[30] D. J. Kedziora, A. Ankiewicz, and N. Akhmediev, Secondorder nonlinear Schrödinger equation breather solutions in the degenerate and rogue wave limits, Phys. Rev. E 85, 066601 (2012).

[31] A. Chowdury, D. J. Kedziora, A. Ankiewicz, and N. Akhmediev, Breather-to-soliton conversions described by the quintic equation of the nonlinear Schrödinger hierarchy, Phys. Rev. E 91, 032928 (2015).

[32] A. Chowdury, A. Ankiewicz, and N. Akhmediev, Moving breathers and breather-to-soliton conversions for the Hirota equation, Proc. R. Soc. A 471, 20150130 (2015).

[33] A. Chowdury and W. Krolikowski, Breather-to-soliton transformation rules in the hierarchy of nonlinear Schrödinger equations, Phys. Rev. E 95, 062226 (2017).

[34] E. A. Kuznetsov, Solitons in a parametrically unstable plasma, Dokl. Akad. Nauk SSSR 236, 575 (1977) [Sov. Phys. Docl. 22, 507 (1977)]. 
[35] Y.-C. Ma, The perturbed plane-wave solutions of the cubic Schrödinger equation, Stud. Appl. Math. 60, 43 (1979).

[36] J.-W. Yang, Y.-T. Gao, C.-Q. Su, Q.-M. Wang, and Z.-Z. Lan, Breathers and rogue waves in a Heisenberg ferromagnetic spin chain or an alpha helical protein, Commun. Nonlin. Sci. Numer. Simulat. 48, 340 (2017).

[37] A. Chowdury, D. J. Kedziora, A. Ankiewicz, and N. Akhmediev, Breather solutions of the integrable quintic nonlinear Schrödinger equation and their interactions, Phys. Rev. E 91, 022919 (2015).

[38] A. Ankiewicz, D. J. Kedziora, and N. Akhmediev, Rogue wave triplets, Phys. Lett. A 375, 2782 (2011).

[39] P. Gaillard, Other $2 N-2$ parameter solutions of the NLS equation and $2 N+1$ highest amplitude of the modulus of the $N$ th order Akhmediev-Peregrine breather, J. Phys. A Math. Theor. 48, 145203 (2015).

[40] P. Y. G. Dontsop, B. G. O. Essama, J. M. Doing, M. M. Dedzo, J. Atangana, D. Yemele, and T. C. Kofane, AkhmedievPeregrine rogue waves generation in a composite right/lefthanded transmission line, Opt. Quant. Electron. 48, 59 (2016).

[41] A. Ankiewicz, P. A. Clarkson, and N. Akhmediev, Rogue waves, rational solutions, the patterns of their zeros and integral relations, J. Phys. A: Math. Theor. 43, 122002 (2010).

[42] N. Akhmediev and A. Ankiewicz, Spatial soliton X-junctions and couplers, Opt. Commun. 100, 186 (1993).

[43] D. J. Kedziora, A. Ankiewicz, and N. Akhmediev, Circular rogue wave clusters, Phys. Rev. E 84, 056611 (2011).

[44] V. E. Zakharov, Stability of periodic waves of finite amplitude on the surface of a deep fluid, J. Appl. Mech. Tech. Phys. 9, 190 (1968).

[45] M. Erkintalo, K. Hammani, B. Kibler, C. Finot, N. Akhmediev, J. M. Dudley, and G. Genty, Higher-Order Modulation
Instability in Nonlinear Fiber Optics, Phys. Rev. Lett. 107, 253901 (2011).

[46] P. Walczak, S. Randoux, and P. Suret, Optical Rogue Waves in Integrable Turbulence, Phys. Rev. Lett. 114, 143903 (2015).

[47] D. S. Agafontsev and V. E. Zakharov, Integrable turbulence and formation of rogue waves, Nonlinearity 28, 2791 (2015).

[48] N. Akhmediev, J. M. Soto-Crespo, and N. Devine, Breather turbulence versus soliton turbulence: Rogue waves, probability density functions, and spectral features, Phys. Rev. E 94, 022212 (2016).

[49] D. Solli, C. Ropers, P. Koonath, and B. Jalali, Optical rogue waves, Nature (London) 450, 1054 (2007).

[50] J. M. Dudley, G. Genty, and S. Coen, Supercontinuum generation in photonic crystal fiber, Rev. Mod. Phys. 78, 1135 (2006).

[51] I. Bialynicki-Birula, On the stability of solitons, in Nonlinear Problems in Theoretical Physics, edited by A. F. Rañada, Lecture Notes in Physics Vol. 98 (Springer, Berlin, 1979).

[52] N. G. Vakhitov and A. A. Kolokolov, Stationary solutions of the wave equation in the medium with nonlinearity saturation, Izv. Vuz. Radiofiz. 16, 1020 (1973) [Radiophys. Quantum Electron. 16, 783 (1973)].

[53] C. Mahnke and F. Mitschke, Possibility of an Akhmediev breather decaying into solitons, Phys. Rev. A 85, 033808 (2012).

[54] A. Calini and C. M. Schober, Numerical investigation of stability of breather-type solutions of the nonlinear Schrödinger equation, Nat. Hazards Earth Syst. Sci. 14, 1431 (2014).

[55] L. Duan, L.-C. Zhao, W.-H. Xu, C. Liu, Z.-Y. Yang, and W.-L. Yang, Soliton excitations on a continuous-wave background in the modulational instability regime with fourth-order effects, Phys. Rev. E 95, 042212 (2017). 